ND

$13 \varnothing 1$

N5N27

C. 2

NPG

\title{
AN EXHIBITION OF
}

\section{PORTRAITS}

FOR THE BENEFIT OF

THE ORTHOPÆDIC HOSPITAL

NATIONAL ACADEMY OF DESIGIV NEW YORK

$$
\text { DECEMBER, } 1898
$$
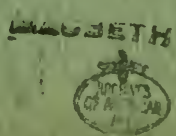


\section{Bonaventure's Gallery,}

\section{West 33d Street, opposite the Waldorf.}

MR. E. F. BONAVENTURE desires to announce to his numerous patrons and the lovers of art his return from abroad with an unusual assortment of its choicest works.

He has on view most beautiful specimens of Books in exquisite bindings, first editions, rare Americana, extra illustrated historical and Court memoirs. Bric-à-brac, gold boxes, miniatures on ivory, antique fans and other art objects. Fine paintings in oil and water color, engravings and etchings of all schools, many artistically framed.

He most cordially solicits a visit to his galleries, feeling assured that the examination of his exhibits for the season $1898-9$ will amply repay either connoisseur or collector.

\section{CRIST DELMONICO, \\ I 66 FIFTH AVENUE,}

BETWEEN 2IST AND 22D STREETS,

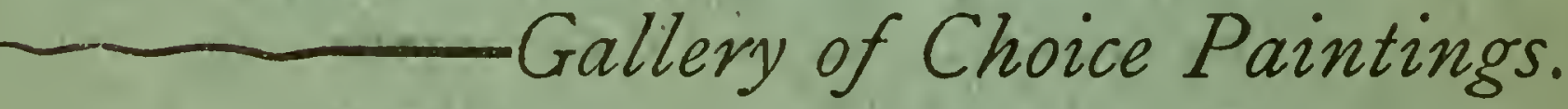




\section{New York}

\section{Orthopœedic Dispensary and Hospital}

Nos. 126 and 128 East 59th Street

Between Fourth and Lexington Aves.

Open from I.30 to 3.30 P.M. (Sundays and Legal Holidays excepted) for the Reception and Treatment of Patients.

Objects of The Institution.- " To furnish treatment to the poor, with special reference to the Diseases and Deformities of the Spine and the Hip Joint, and other of tire more serious diseases of the bones and joints requiring surgical and mechanical treatment, and for giving instruction in the same." All cases of defurmity besides those mentioned, including Club-Foot, Lateral Curvature of the Spine, Infantile Paralysis, Bow-Legs, \&c., are received and treated.

The Medical Profession and the public generally are invited to visit the institution.

'The Institution is supported by the contribution of the henevolent. Contributions, which are earnestly solicited, may be sent to JAS. K. Gracie, Treasurer, 8o Broadway.

\section{OFFICERS AND TRUSTEES}

O. EGERTON SCHMIDT

President

EDWARD A. SMITH

Treasurer

LOUIS V. BRIGHT

Secretary

J. K. GRACIE

Chairman Exec. Committee 


\section{KNOEDLER \& CO.}

\section{Paintings

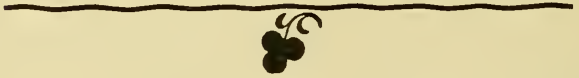

By Deceased and Modern Masters

of the various Schools

迎?

\section{WATER COLORS}

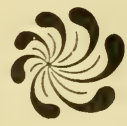

PRINTS

FRAMES

355 Fifth Avenue

Cor. 34th Street,

NEW YORK 
ND

1301

N5N27

${ }^{C 2} \mathrm{LPG} \mathrm{LAN}$

EXHIBITION

OF

PORTRAITS

FOR THE BENEFIT OF

THE ORTHOPÆDIC HOSPITAL

FROM THE FOURTEENTH OF DECEMBER, I 898

TO THE FOURTEENTH OF JANUARY, I 899

NMAAINPG LIBRARY

AUG in lisey

SMITHSONIAN INSTITUTION

NATIONAL ACADEMY OF DESIGN

CORNER OF FOURTH AVE. AND TWENTY-THIRD ST.

NEW YORK . 
The Committee desires to express its thanks to THE NEW YoRK Telephone Company for courtesies extended. 


\section{EXECUTIVE COMMITTEE:}

CHARLES T. BARNEY, ChAIRMAN, E. HAMILTON BELL, SEcretary, FRED. G. KING, Asst. Secretary, EDWARD A. SMITH, TREASURER, HENRY MARQUAND, PRESCOTT HALL BUTLER, STANFORD WHITE, JAMES L. BREESE, ROBERT WALLER, JR.

\section{LADIES' COMMITTEE :}

Mrs. JOHN HOBART WARREN, Chatrman, MrS. CHARLES B. ALEXANDER, Mrs. R. FULTON CUTTING, MRS. EDMUND BAYLIES, MRS. M. DWIGHT COLLIER, Miss DE FOREST, Miss WHITNEY.

\section{ARTISTS' COMMITTEE :}

CHARLES T. BARNEY, CHAIRMAN, J. CARROLL BECKWITH, WILLIAM M. CHASE, EDWIN H. BLASHFIELD, BENJAMIN C. PORTER, STANFORD WHITE, FRANCIS LATHROP.

MINIATURES :

DOUGLAS JOHN CONNAH. 


\section{LIST OF PATRONESSES.}

Mrs. ABERCROMBIE.

Miss F. ADAM.

MRS. EDWARD D. ADAMS.

MRS. THATCHER M. ADAMS.

Mrs. CHARLES B. ALEXANDER.

Mrs. WILLIAM ALEXANDER.

MRS. HENRY ALEXANDRE.

MRS. JOHN E. ALEXANDRE.

Mrs. ASTOR.

MRS. JOHN JACOB ASTOR.

MRS. ROBERT BACON.

MRs. GEORGE F. BAKER.

MRS. CHAS. T. BARNEY.

Mrs. BAYLIES.

Mrs. EDMUND L. BAYLIES.

MRS. F. H. BENEDICT.

MISS HELEN BENEDICT.

MRS. JAMES BENEDICT.

MRS. S. N. BENJAMIN.

MRS. BERWIND.

MRS. FREDERIC BETTS.

MRS. GEORGE BIRD.

MRS. DAVID WOLFE BISHOP.

MRS. HEBER BISHOP.

MRS. WILLIAM METCALF BLISS.

MRS. PENDLETON BOWLER.

MISS BREESE.

MRS. BENJAMIN BREWSTER.

MRS. CALVIN S. BRICE.

MRS. LOUIS V. BRIGHT.

MRS. MORTIMER BROOKS.

MRS. LLOYD BRYCE.

MRS. W. LANMAN BULL.

MRS. I. TOWNSEND BURDEN.

MRS. JAMES ABERCROMBIE BURDEN.

Mrs. McCOSKRY BUTT. 
Mrs. PRESCOTT HALL BUTLER.

Miss CALLENDER.

MRS. ADDISON CAMMACK.

Mrs. A. CASS CANFIELD.

MRS. AMORY SIBLFY CARHART.

MRS. CARNEGIE.

MRS. LINDLEY HOFFMAN CHAPIN.

MRS. CLAPP.

MRS. HENRY CLEWS.

MRS. STUART COATS.

MRS. EDMUND COFFIN.

Mrs. M. DWIGHT COLLIER.

Mrs. CHARLES H. COSTER.

MRS. HENRY A. COSTER.

MRS. W. S. COWLES.

Mrs. GEORGE CROCKER.

MRS. WM. BAYARD CUTTING.

Mrs. R. FULTON CUTTING.

MISS CUYLER.

Mrs. JOHN DAVIS.

Miss C. DE FOREST.

Mrs. GEO. B. DE FOREST.

MRS. LOCKWOOD DE FOREST.

MRS. ROBERT W. DE FOREST.

Miss DEHON.

MRS. RICHARD DELAFIELD.

Mrs. EUGENE DELANO.

MRS. WM. B. DENSMIORE, JR.

MRS. CHARLES DE RHAM.

MRS. CHAS. DE RHAM, JR.

Mrs. H. CASIMIR DE RHAM.

MRs. GEO. G. DE WITT.

MRS. ARTHUR DODGE.

MRS. CLEVELAND DODGE.

Mrs. GEO. DODGE.

MrS. WM. E. DODGE, JR.

MrS. GEO. WM. DOUGLAS.

MRS. JOHN BEVERLEY DUER.

MRS. JOHN P. DUNCAN.

Mrs. ELISHA DYER, JR.

Mrs. ALFRED ELY. 
MRS. HENRY EMMET.

MRS. STUYVESANT FISH.

MrS. JOSIAH M. FISKE.

MRS. LOUIS FITZGERALD.

Mrs. FREDERICK FOSTER.

Mrs. GIRAUD FOSTER.

MRS. CHARLES FRANCKLYN.

MRS. KENNETH FRAZIER.

MRS. THEODORE FRELINGHUYSEN.

MRS. SETH BARTON FRENCH.

MIsS C. FURNISS.

Mrs. ELBRIDGE T. GERRY.

MrS. GEORGE R. GIBSON.

Mrs. JAMES A. GLOVER.

MRS. J. WARREN GODDARD.

MRS. ROBERT GOELET.

Mrs. GEORGE J. GOULD.

Miss GOULD.

MISS HELEN GOULD.

MRS. MALCOLM GRAHAM.

MRS. JOHN GREENOUGH.

Mrs, CHESTER GRISWOLD.

MIss GURNEE.

MrS. W. D. GUTHRIE.

MRS. HAROLD F. HADDEN.

MRS. J. E. SMITH HADDEN.

Mrs. JOHN A. HADDEN, JR.

Mrs. J. B. HAGGIN.

MrS. F. R. HALSEY.

Miss E. S. HAMILTON.

MRS. J. MONTGOMERY HARE.

MRS. J. BORDEN HARRIMAN.

MRS. CHARLES R. HENDERSON.

MADAME HEREDIA.

MRS. E. H. HERRIMAN.

Mrs. HEWITT.

Miss S. C. HEWITT.

MRS. MOSES HOPKINS.

MRS. HENRY E. HOWLAND.

Mrs. GERALD L. HOYT.

MRS, LOUIS HOYT. 
Miss ISELIN.

Mrs. ADRIAN ISELIN, JR.

Mrs. C. OLIVER ISELIN.

MRS. COLUMBUS O'D. ISELIN

Mrs. WILLIAM ISELIN.

MRS. BRAYTON IVES.

MRS. HENRY A. JAMES.

MRS. WALTER JENNINGS.

MRS. MORRIS K. JESUP.

MRS. BRADISH JOHNSON.

MRS. S. FISHER JOHNSON.

MRS. JOHN D. JONES.

MRS. LOUIS COLFORD JONES.

Mrs. JUILLIARD.

MRs. GRENVILLE KANE.

MRS. J. FREDERIC KERNOCHAN.

MRS. KIDD.

Mrs. EDWARD KING.

MRS. PERCY KING.

Mrs. F. P. KINNICUTT.

MRS. KORTRIGHT.

MrS. WOODBURY LANGDON.

Miss LEARY.

MrR. E. LIVINGSTON LUDLOW.

MRS. DANIEL LORD.

MRS. LORILLARD.

Mrs. PIERRE LORILLARD, JR.

MRS. RICHARD LOUNSBERY.

MRS. SETH LOW.

MRS. EDW. L. LUDLOW.

MRS. CLARENCE H. MACKAY.

Mrs. PIERRE MALI.

Mrs. WILLIAM MANICE.

MRS. HENRY MARQUAND.

Miss LOUISE W. MCALLISTER.

Mrs. JOHN J. McCOSH.

Mrs. J. G. McCULloUGH.

Mrs. HENRY METCALFE.

MRS. JOSEPH MILLBANK.

MRS. WM. STARR MILLER.

Mrs. OGDEN MILLS. 
MRS. MINTURN.

MRS. R. BURNHAM MOFFAT.

MRS. JULES A. MONTANT.

MRS. CASIMIR DE RHAM MOORE.

MRS. ANSON B. MORAN.

MRS. DANIEL E. MORAN.

MRS. J. PIERPONT MORGAN.

MRS. JUNIUS MORGAN.

MRS. DAVID MORRISON.

MRS. STANLEY MORTIMER.

MRS. LEVI P. MORTON.

MRS. JOHN G. NEESER.

Mrs. NEILSON.

Miss NELSON.

MISS NORRIE.

MRS. OELRICHS.

MRS. HENRY FAIRFIELD OSBORN.

MRS. WM. CHURCH OSBORN.

Mrs. WILLIAM H. OSGOOD.

Mrs. HUGH ALMERIC PAGET.

MRS. HENRY PARISH, JR.

MRS. TRENOR L. PARK.

MRS. H. DE B. PARSONS.

MRS. HERBERT PELL.

MRS. F. K. PENDLETON.

MRS. WILLIAM A. PERRY.

MRS. CHARLES G. PETERS.

Mrs. EDWARDS PIERREPONT.

MRS. WM. POLLOCK.

MRS. BENJAMIN PORTER.

MISS GRACE HOWARD POTTER.

MRS. EDMUND RANDOLPH.

MRS. EDW. S. RAPELLO.

MRS. GERALDYN REDMOND.

MRS. ROLAND REDMOND.

MRS. WHITELAW REID.

Miss ELIZABETH REMSEN.

Mrs. GEO. L. RIVES.

Mrs. REGINALD RIVES.

MRS. J. HAMPDEN ROBB.

MRS. DOUGLAS ROBINSON, JR. 
MRS. JOHN A. ROBINSON. Mrs. WILLIAM ROBISON.

MRS. BURKE ROCHE.

MRS. WM. ROCKEFELLER.

MRS. HENRY ROGERS.

Mrs. C. V. S. ROOSEVELT.

Mrs. JAMES ROOSEVELT.

MRS. JOHN A. ROOSEVELT.

MRS. HENRY SANDERS.

Mrs. B. AYMAR SANDS.

MRS. EUGENE SCHIEFFELIN.

MRS. WM. JAY SCHIEFFELIN.

Mrs. OSCAR EGERTON SCHMIDT.

MRS. WINFIELD SCOTT.

MRS. ROBERT SEDGWICK.

MRS. FREDERICK SHELDON.

MrS. ELLIOTT F. SHEPARD.

MRS. WM. WATTS SHERMAN.

MRS. HENRY SLOANE.

MRS. SAMUEL SLOAN.

Mrs. SAMUEL SLOAN, JR,

Mrs. JOHN SLOANE.

Mrs. WM. D. SLOANE.

Mrs. E. A. SMITH.

MisS JOSEPHINE C. SMITH.

MRS. WALKER BREESE SMITH.

MrS. JAMES RUSSELL SOLEY.

MRS. VICTOR SORCHON.

MRS. JAMES SPEYER.

MRS. WM.RHINELANDER STEWART.

MRS. JOSEPH STICKNEY.

Mrs. ANSON PHELPS STOKES.

Miss C. PHELPS STOKES.

MRS. I. N. P. STOKES.

Mrs. W. E. D. STOKES.

MRS. JULIAN STORY.

MRS. MARION STORY.

MRS. WILLIAM E. STREET.

MRS. W. EVERARD STRONG.

MRS. W. L. STRONG.

MRS. WALTER L. SUYDAM. 
MRS. SAMUEL THOMAS.

Mrs. FREDERICK F. THOMPSON.

MRS. J. TODHUNTER THOMPSON.

MRS. DAVID THOMSON.

MRS. LOUIS C. TIFFANY.

MRS. J. KENNEDY TOD.

MRS. PAUL TUCKERMAN.

MISS KATHERINE E. TURNBULL.

Mrs. H. McK. TWOMBLY.

MRS. VANDERBILT.

MRS. CORNELIUS VANDERBILT, JR.

MRS. FREDERICK VANDERBILT.

MRS. JOSEPH WALKER, JR.

Mrs. ROBERT WALLER, JR.

MRS. GEO. HENRY WARREN.

MRS. JOHN HOBART WARREN.

Miss E. A. WATSON.

MRS. WALTER WATSON.

Mrs. ALEXANDER S. WEBB.

Mrs. H. WALTER WEBB.

MRS. W. STORRS WELLS.

Miss CAROLINE WHITE.

Miss WHITNEY.

Mrs. HY. PAYNE WHITNEY.

Mrs. WM. C. WHITNEY.

Mrs. WILLIAM WHITNEY.

Mrs. JOHN C. WILMERDING.

MRS. ORME WILSON.

MRS. EDWARD WINSLOW.

MRS. BUCHANAN WINTHROP.

MRS. FRANCIS DE R. WISSMAN.

MRS. FRANK WITHERBEE.

Mrs. FRANK S. WITHERBEE.

MRS. JOHN M. WOODBURY.

MRS. JAMES A. WRIGHT.

Mrs. WYSONG. 


\title{
CATALOGUE.
}

\section{PORTRAITS.}

\author{
J. C. ARTER. (American.)
}

I. Mrs. J. C. Wysong.

Loaned by J. C. ARTER.

2. Mrs. Francis Burrall Hoffman.

Loaned by J. C. ARTER.

3. Miss Austin.

Loaned by J. C. ARTER.

\section{F. ALEXANDER. (American.)}

4. Mrs. Henry F. French.

Loaned by D. C. FRENCH. 
JOHN W. ALEXANDER. (American.)

5. James W. Alexander.

Loaned by J. W. ALEXANDER.

6. Joseph Jefferson.

Loaned by J. W. ALEXANDER.

7. Mrs. B.

Loaned by J. W. ALEXANDER.

8. Miss V.

Loaned by J. W. ALEXANDER.

9. A Child.

Loaned by J. W. ALEXANDER.

\section{A. A. ANDERSON. (American.)}

Io. Mrs. Arthur Kemp.

Loaned by A. A. ANDERSON.

II. Mrs. S. L. Anderson.

Loaned by' A. A. ANDERSON.

12. Mrs. Francis P. Kinnicutt.

Loaned by A. A. ANDERSON.

\section{MAJOR ANDRÉ. (English.)}

13. Portrait of Himself.

Loaned by J. W. BOUTON. 
MARIA BROOKS. (American.)

I4. Mrs. H. B.

Loaned by MARIA BROOKS.

I5. Master L. C. F.

Loaned by PAUL FULLER.

\section{BONNAT. (French.)}

I6. Wm. A. Duer.

Loaned by W. A. DUER.

I7. Hon. Abram S. Hewitt.

Loaned by Mrs. A. S. HEWITT.

FRANK W. BENSON. (American.)

I8. Mrs. P. K.

Loaned by PERCIVAL KNAUTH.

\section{G. BOLDINI. (Italian.)}

19. Mrs. Henry W. Poor.

Loaned by H. W. POOR.

20. Portrait of a Child.

Loaned by WM. H. CROCKER.

21. Miss Elsie de Wolfe.

Loaned by MISS DE WOLFE. 
CECELIA BEAUX. (American.)

22. Mother and Son.

Loaned by Mr. BEAUVEAU BORIE.

23. Portrait of Cynthia.

Loaned by MRS. A. M. SHERWOOD.

24. Dorothea and Francesca.

Loaned by CECELIA BEAUX.

EDWARD A. BELL. (American.)

25. Portrait of Mrs. C.

Loaned by EDWARD A. BELL.

SIR WM. BEECHY, R. A., I753-I839. (English.)

26. Miss Harriet Douglas.

Loaned by MRS. DOUGLAS ROBINSON.

27. Family Group. (James Lorimer.)

Loaned by MALCOLM GRAHAM.

\section{GEO. H. BAKER. (American.)}

28. Hon. B. Parke Avery.

Loaned by SAMUEL P. AVERY. 
J. CARROLL BECKWITH. (American.)

29. Col. Cannon.

Loaned by MRs. CHESTER GRISWOLD.

30. Miss Polly Ogden.

Loaned by MRS. DAVID B. OGDEN.

3I. Mrs. Beckwith.

Loaned by J. CARROLL BECKWITH.

32. Miss Florence Robinson.

Loaned by J. CARROLL BECKWITH.

33. Portrait.

Loaned by J. CARROLL BECKWITH.

34. Portrait.

Loaned by J. CARROLL BECKWITH.

\section{W. BOUGUEREAU. (French.)}

35. Portrait of a Boy.

Loaned by MrS. DAVID WOLFE BISHOP.

CARL J. BLENNER. (American.)

36. Miss Catharine Gill.

Loaned by CARL J. BLENNER. 
SANDRO BOTTICELLI, I446-I510. (Italian.)

37. Portrait of Himself.

Loaned by JOHN J. JOHNSON.

\section{T. CHARTRAN.}

38. Mrs. Clarence H. Mackay.

Loaned by CLARENCE H. MACKAY.

39. A Portrait.

Loaned by TRENOR L. PARK.

40. Mrs. Theodore Frelinghuysen.

Loaned by T. FRELINGHUYSEN.

4I. Mrs. Jules A. Montant.

Loaned by JULES A. MONTANT.

42. Charles B. Alexander.

Loaned by C. B. ALEXANDER.

43. Hamilton Fish.

Loaned by HAMILTON FISH.

44. A Portrait.

Loaned by Mrs. W. D. SLOANE.

J. WELLS CHAMPNEY. (American.)

45. Mrs. Wright P. Edgerton.

Loaned by Mrs. WM. TOD HELMUTH. 
46. Miss Caroline L. Goodridge.

Loaned by C. M. GOODRIDGE.

47. Miss Winslow (Mrs. Arthur W. Little).

Loaned by EDWARD WINSLOW.

\section{ALEXANDRE CABANEL. (French.)}

48. John W. Mackay.

Loaned by MRS. CLARENCE H. MACKAY.

49. Portrait of a Lady.

Loaned by AMEDIE DEPAU MORAN.

\section{ALFRED COLLINS. ' (American.)}

50. Portrait of W. S. R.

Loaned by REv. W. S. RAINSFORD, D.D.

5I. Dr. William H. Draper.

Loaned by THE SOCIETY OF THE NEW YORK HOSPITAL.

\section{LEON COMERRE. (French.)}

52. Countess de Laugier Villars.

Loaned by JOHNSTON LIVINGSTON.

53. Mrs. G. Redmond.

Loaned by JOHNSTON LIVINGSTON.

54. Johnston and Roland L. Redmond.

Loaned by JOHNSTON LIVINGSTON. 
BENJAMIN CONSTANT. (French.)

55. Mrs. Eugene W. Glaenzer.

Loaned by Mrs. EUGENE W. GLAENZER.

56. Mrs. Wm. R. Thompson.

Loaned by W.M. R. THOMPSON.

57. Wm. R. Thompson.

Loaned by WM. R. THOMPSON.

CHAS. C. CURRAN. (American.)

58. Mrs. Lydia S. Woodworth. First President of the Lake Erie Seminary.

Loaned by CHAS. C. CURRAN.

59. Mrs. Walter L. Palmer.

Loaned by CHAS. C. CURRAN.

6o. Dr. Eugene Coleman Savidge.

Loaned by EUGENE COLEMAN SAVIDGE.

\section{FRANCIS COTES. (English.)}

6r. The Artist.

Loaned by JULIUS OEHME.

\section{WILLIAM M. CHASE. (American.)}

62. Mrs. Geo. B. Ely.

Loaned by Mrs. G. B. ELY. 
63. J. Henry Harper.

Loaned by J. HENRY HARPER.

64. Miss C.

Loaned by' MRS. C.

65. Miss F. De Forest.

Loaned by Mrs. ROBT. W. DE FOREST.

66. Mona.

Loaned by MRS. W. MONCURE ROBINSON.

67. Miss X.

Loaned by WM M. CHASE.

J. S. COPLEY. (Amcrican.)

68. Governor Colden and his Grandson Warren De Lancey.

Loaned by H. A. COSTER.

69. Mrs. Ralph Izzard (Alice De Lancey).

Loaned by H. A. COSTER.

70. John A. Graham, LL.D.

Loaned by MALCOLM GRAHAM. 


\section{GEORGINE CAMPBELL. (American.)}

7I. Mrs. J. Vinton Dahlgren.

Loaned by JOHN VINTON DAHLGREN.

72. Portrait of a Boy.

Loaned by Mrs. W. A.

K. HOLSTON CARPENTER. (American.)

73. Mrs. Louis Steiner and Sons.

Loaned by' K. HOLSTON CARPENTER.

ANNIBALE CARRACCI, I560-1609. (Italian.)

74. Portrait.

Loaned by ROBT. W. DE FOREST.

\section{KENYON COX. (American.)}

75. Portrait of a Young Man.

Loaned by KENYON COX.

76. Profile of Mrs. O.

Loaned by L. C. OPDYCKE.

77. Susie (aged 7).

Loaned by PRESCOTT HALL BUTLER.

CHAS. MELVILLE DEWEY. (American.)

78. Rev. Canon St. John of Gloucester Cathedral. Loaned by CHAS. MELVILLE DEWEY. 


\section{CAROLUS DURAN. (French.)}

79. Portrait of a Lady.

Loaned by Mrs. JOHN SLOANE.

8o. Portrait of a Lady.

Loaned by MRS. JOHN SLOANE.

8I. Miss Morton.

Loaned by HON, LEVI P. MORTON.

82. Johnston Livingston.

Loaned by KNICKERBOCKER CLUB.

83. Lady Terence Blackwood.

Loaned by JOHN H. DAVIS.

T. W. DEWING. (American.)

84. Portrait of a Lady.

Loaned by T. W. DEWING.

\section{DAGNAN-BOUVERET. (French.)}

86. Geo. F. Baker.

Loaned by GEO. F. BAKER.

87. Mme. Dagnan-Bouveret in Bernaise Costume. Loaned by JOHN J. JOHNSON. 


\section{PERCIVAL DE LUCE. (American.)}

88. Mrs. John Irving.

Loaned by PERCIVAL DE LUCE.

\section{A. DE LA GANDARA. (French.)}

89. Mrs. Burke Roche.

Loaned by DURAND RUEL.

90. Portrait of a Child.

Loaned by P. SPAULDING.

JACQUES L. DAVID, I748-I825. (French.)

91. Mrs. Moeran.

Loaned by Mrs. HENRY LE BARBIER.

RAIMUNDO DE MADRAZO. (Spanish.)

92. Duchesse de Morny.

Loaned by JULIUS OEHME.

93. Dr. W. M. Polk.

Loaned by DR. POLK.

94. Mrs. Oliver G. Jennings.

Loaned by MRS. JENNINGS. 
95. Miss Schermerhorn.

Loaned by MRS. KANE.

96. Mrs. Kane.

Loaned by MRS. KANE.

VAN DE MEER DE DELFT, I632-I675. (Dutch.)

97. Woman with Guitar.

Loaned by JOHN J. JOHNSON.

GERARD DOUW, I6I3-1675. (Dutch.)

98. Lady Playing a Virginal.

Loaned by GEO. J. GOULD.

P. DE LA FRANCESCA, I4I6-I492. (Italian.) 99. Profile of Woman.

Loand by JOHN J. JOHNSON.

LYDIA FIELD EMMET. (American.)

ıоO. Mrs. Wm. Hamilton Russell.

Loaned by WM. HAMILTON RUSSELL.

IOI. Frederick Stevens Allen.

Loand by F. S. ALLEN.

IO2. Master John Wells.

Loaned by MRS. O. O. WEL.LS. 
WYATT EATON. (American.)

I03. Minna Craven de Kay.

Loaned by MRS. DE KAY.

KENNETH FRAZIER. (American.)

I04. Portrait of Child.

Loaned by KENNETH FRAZIER.

I05. Mrs. Samuel Sloane, Jr.

Loaned by SAMUEL SLOANE, JR.

JARED B. FLAGG. (American.)

106. Mrs. W. Allston Flagg.

Loaned by J. B. FLAGG.

\section{FRANK FOWLER. (American.)}

107. John D. Crimmins.

Loaned by JOHN D. CRIMMINS.

I08. Mrs. B.

Loaned by MRS. F. CADWALADER BREWSTER.

I09. Miss $\mathrm{H}$.

Loaned by FRANK FOWLER. 
GEORGE FULLER. (American.)

I IO. Mrs. Isaac Marquand.

Loaned by HENRY MARQUAND.

\section{F. P. FINOCCHIARO. (Italian.)}

I I I. Mme. Bjorksten.

Loaned by FRANCESCO P. FINOCCHIARO.

W. H. FUNK. (German.)

I I Mrs. Clinch Smith.

Loaned by W. H. FUNK.

J. L. GEROME. (French.)

II 3. Mrs. Truax.

Loaned by JUDGE CHAS. H. TRUAX.

THOMAS GAINSBOROUGH, I727-1788.

(English.)

II4. Blue Boy.

Loaned by GEO. A. HEARN.

II5. The Artist.

Loaned by MRS. WM. T. BLODGETT. 
I I6. Portrait of Geo. Coyte.

Loaned by JOHN J. JOHNSON.

I I 7. Master Jack Hill.

Loaned by GEO. J. GOULD.

II8. Lady Musgrave.

Loaned by GEO. J. GOULD.

WM. H. HYDE. (American.)

I I9. Mrs. C. H. Russell and Children.

Loaned by CHAS. H. RUSSELL.

I20. Miss J. C. Reid.

Loaned by MRs. REID.

I2 I. Miss Cora Randolph.

Loaned by MRS. RANDOLPH.

JOHN HOPPNER, I758-1810. (English.)

122. Portrait of a Lady.

Loaned by C. P. HUNTINGTON.

I23. Dancing Girl.

Loaned by HARRY PAYNE WHITNEY.

I24. Boy and Dog.

Loaned by JULIUS OEHME.

I25. Ideal Head.

Loaned by JULIUS OEHME, 


\section{HUNTINGTON. (American.)}

I26. Martin van Buren, President U. S.

Loaned by' D. HUNTINGTON.

127. Mrs. Leopold H. Francke.

Loaned by D. HUNTINGTON.

128. Miss Marie Huntington.

Loaned by D. HUNTINGTON.

WILLIAM MCLURE HAMILTON. (American.)

I29. Right Hon. Wm. E. Gladstone, of Downing Street.

Loaned by THE PENNSYLVANIA ACADEMY OF THE FINE ARTS.

ROBERT GORDON HARDIE. (American.)

I30. Mrs. Robt. M. Morse.

Loaned by ROBT. GORDON HARDIE.

I31. Edward A. Strong.

Loand by ROBT. GORDON HARIIE.

FRANK HOLL, R. A. (English.)

132. Edward D. Adams.

Loaned by EDWARD D. ADAMS.

I33. J. Pierpont Morgan.

Loaned by J. P. MORGAN. 
ALBERT HERTER. (American.)

I34. Mathilde-Baroness de R.

Loaned by ALBERT HERTER.

\section{EDWARD HUGHES. (English.)}

135. Miss Helen Morton.

Loaned by LEVI P. MORTON.

136. Miss Lena Morton.

Loaned by LEVI P. MORTON.

E. M. HELLER. (American.)

I37. Mrs. H. T. Seymour (née Damrosch).

Loaned by E. M. HELLER.

WILLIAM HOGARTH, I697-I767. (English.)

I38. Portrait of a Man.

Loaned by S. P. AVERY.

LOUISA L. HEUSTIS. (American.)

139. V. C. Reynal.

Loaned by L. L. HEUSTIS. 


\section{S. S. HENNER. (French.)}

140. Woman's Head.

Loaned by Mrs. CHAS. B. ALEXANDER.

S. ISHAM. (American.)

I4I. Miss C. H.

Loaned by S. ISHAM.

I42. Mrs. C.

Loaned by $\mathrm{S}$. ISHAM.

HENRY INMAN, I8OI-1846. (American.)

I43. President Van Buren.

Loaned by WM. A. DUER.

I44. Wm. A. Duer, President Columbia College.

Loaned by WM. A. DUER.

EASTMAN JOHNSON. (American.)

I45. Mrs. Alfred R. Conkling.

Loaned by A. R. CONKI.ING.

JOHN WESLEY JARVIS, I780-I846.

(American.)

146. Commodore Perry.

Loaned by MRS. HENRY F. OSBORN. 
CORNELIUS JANSEN, I590-I665. (Dutch.)

I47. Lord Fairfax.

Loaned by MRS. FREDERIC BRONSON

SUSAN M. KETCHAM. (American.)

I48. Mrs. Ketcham.

Loaned by Miss S. M. KETCHAM.

DORA W. KEITH. (American.)

I49. Portrait of a Child.

Loaned by DORA WHEELER KEITH.

I50. Portrait of my Mother.

Loaned by DORA WHEELER KEITH.

WILLIAM SARGEANT KENDALL.

(American.)

I5 I. Portrait of a Child.

Loaned by WM. SERGEANT KENDALL.

I 52. Miss Chanler.

Loaned by Miss CHANLER.

FRANCIS LATHROP. (American.)

I53. Unfinished Portrait.

Loaned $b y$ FRANCIS LATHROP. 
SIR PETER LELY, I6I7-1680. (English.)

I54. Mrs. Claypoole.

Loaned by Mrs. CORNELIUS VANDERBILT.

\section{SIR THOMAS LAWRENCE, I769-1830.}

(English.)

155. Portrait of Two Children.

Loaned by H. PAYNE WHITNEY.

156. Portrait of a Lady.

Loaned by J. C. HOAGLAND.

I57. John Philip Kemble.

Loaned by MRS. OIVEN WISTER.

I58. Lady Bathurst.

Loaned by JULIUS OEHME.

JOHN LAMBERT, JR. (American.)

I 59. Dorothy Harrison.

Loaned by MRS. CHAS. C. HARRISON.

WM. H. LIPPINCOTT. (American.)

I60. Mrs. Wm. J. Le Moyne.

Loaned by Mrs. WM. J. LE MOYNE. 
NICHOLAS LARGILLIERE, I656-I746.

(French.)

16I. Duchesse de Villare.

Loaned by C. P. HUNTINGTON.

\section{FANTIN LATOUR. (French.)}

162. J. McN. Whistler.

Loaned by SAMUEL P. AVERY.

E. CHRISTINE LUMSDON. (American.)

I63. E. W. Bliss.

Loaned by E. CHRISTINE LUMSDON.

PAUL MOREELSE, I57I-I688. (Dutch.) 164. Portrait.

Loaned by ROBT. W. DE FOREST.

H. SIDDONS MOWBRAY. (American.)

I65. Mrs. Mowbray.

Loaned by H. SIDDONS MOWBRAY.

I66. Mrs. C. Graves.

Loaned by $\mathrm{H}$. SIDDONS MOWBRAY.

167. Lady in Black.

Loaned by H. SIDDONS MOWBRAY. 


\section{GEO. C. MUNZIG. (American.)}

I68. Mrs. Watts Sherman.

Loaned by GEO. C. MUNZIG.

169. Miss Irene Watts Sherman.

Loaned by GEO. C. MIUNZIG.

I70. Miss Gertrude Watts Sherman.

Loaned by GEO. C. MUNZIG.

I7 I. Mrs. Harriet L. Johnson.

Loaned by' GEO. C. MUNZIG.

I72. Mrs. Stuyvesant Fish.

Loaned by GEO. C. MUNZIG.

MISS JANE MORGAN. (American.)

173. Mrs. Hammond.

Loancd by MISS JANE MORGAN.

GEORGE MORLAND, 1764-1806. (English.)

174. Town.

Loaned by MORRIS K. JESUP.

175. Country.

Loaned by MORRIS K. JESUP.

JEAN MARC NATTIER, 1685-1766. (French.)

176. Empress Elizabeth of Russia.

Loaned by E. J. BERWIND. 
J. OPIE, R. A., I76I-I807. (English.)

I77. Dr. Walcott (Peter Pindar).

Loaned by ARTHUR TOOTH \& SONS.

ORRIN PARSONS. (American.)

I78. Parke Godwin.

Loaned by ORRIN PARSONS.

HARPER PENNINGTON. (American.)

I79. Mrs. Royal Phelps Carroll.

Loaned by HARPER PENNINGTON.

I80. Miss Beatrix Jones.

Loaned by HARPER PENNINGTON.

I8I. Mrs. Lincklaen.

Loaned by CHAS. T. FAIRCHILD.

I82. Wm. H. Beard.

Loaned by HARPER PENNINGTON.

BENJAMIN C. PORTER. (American.)

I83. Mrs. Harry Payne Whitney. (Decorative Panel.)

Loaned by C. VANDERBILT. 
184. Mrs. Chas. B. Alexander.

Loaned by C. B. ALEXANDER.

185. Honorable Mrs. Herbert F. Eaton.

Loancd by F. O. FRENCH.

I86. Mrs. Lloyd Bryce.

Loaned by LLOYD BRYCE.

I87. Miss Marie Winthrop.

Loaned by BUCHANAN WINTHROP.

I88. Mrs. John E. Alexandre.

Loaned by JOHN E. ALEXANDRE.

I89. Mrs. Nathan A. Baldwin.

Loaned by MRs. LORILLARD SPENCER.

190. Mrs. Porter.

Loaned by MR. PORTER.

H. R. POORE. (American.)

191. Mrs. Chas. Emory Stevens.

Loaned by H. R. POORE.

SIR HENRY RAEBURN, 1756-1823. (English.) 192. Portrait of a Boy.

Loaned by C. P. HUNTINGTON. 


\section{WM. J. RICE. (American.)}

193. Portrait of a Man.

Loaned by WM. J. RICE.

194. Portrait of a Lady.

Loaned by WM. J. RICE.

I95. Portrait of a Child.

Loaned by WM. J. RICE.

SIR JOSHUA REYNOLDS, P. R. A., I723-I792.

$$
\text { (English.) }
$$

196. Sachini.

Loaned by BOUSSOD, VALADON \& CO.

197. Lady Stanhope.

Loaned by H. G. MARQUAND.

I98. Portrait of a Lady.

Loaned by HARRY PAYNE WHITNEY.

199. Mrs. Knapp.

Loaned by J. G. MOORE.

200. Lord Stirling.

Loaned by JOHN DUER.

20I. Portrait of Mr. Musters.

Loaned by BLAKESLEE GALLERY. 
202. Miss Waddilove.

Loaned by ARTHUR TOOTH \& SONS.

203. The Strawberry Girl.

Loaned by' GEO. J. GOULI).

DANTE GABRIEL ROSSETTI, I82S-I882.

(English.)

204. Portrait of his Wife.

Loaned ly J. IV. BOUTON.

ROMNEY, R. A., 1734-1802. (English.)

205. Lady with a Muff.

Loaned by H. G. MARQUAND.

206. Mr. Hammond.

Loaned by DURAND RUEL.

207. Mrs. Ralph Willett.

Loaned by DURAND RUEL.

20S. Mrs. Bonar and her Daughter Agnes.

Loaned by KNOEDLER \& CO.

209. Mrs. Fitzherbert.

Loaned by MrS. WM. T. BLODGET'T. 
REMBRANDT VAN RYN, 1607-1669.

2 Io. Portrait.

Loaned by ROBT. W. DE FOREST.

ROSINA EMMET SHERWOOD. (American.)

21 1. Portrait of a Lady.

Loaned by Mrs. ARTHUR M. SHERWOOD.

212. Head.

Loaned by MRS. ARTHUR M. SHERWOOD.

213. Girl and Boy.

Loaned by MRS. ARTHUR M. SHERWOOD.

2I4. Boy.

Loaned by MRs. ARTHUR M. SHERWOOD.

215. Children.

Loaned by MRS. ARTHUR M. SHERWOOD.

216. Boy.

Loaned by MRS. ARTHUR M. SHERWOOD.

217. Brothers.

Loaned by MRS. ARTHUR M. SHERWOOD.

JOHN S. SARGENT, R. A. (American.)

218. Hon. Calvin S. Brice.

Loaned by CALVIN S. BRICE. 
219. Portrait.

Loaned by X.

220. A. Wertheimer.

Loaned by' JOHN S. SARGENT.

22 I. Portrait.

Loaned by MRS. HAROLD F. HADDEN.

\section{JULIAN STORY. (American.)}

222. Miss de Forest.

Loaned by Miss CALLENDER.

GILBERT STUART, I755-1828. (American.)

223. Rufus King.

Loaned by' FRED'K G. KING.

224. Augustin Hicks Lawrence.

Loaned by MISS BREESE.

225. Mrs. Thomas Lea (née Sally Shippen).

Loaned by REV. LEA LUQUER.

226. Senator Robert Young.

Loaned by THOS. B. CLARKE. 


\section{HOBART STRICKLAND. (American.)}

227. Mary S. Kuhn.

Loaned by C. HOBART STRICKLAND.

JULES STEWART. (American.)

228. Mrs. Victor Sorchon.

Loaned by VICTOR SORCHON.

A. D. STURDEVANT. (American.)

229. Mrs. Elisha Dyer.

Loaned by Mrs. DYER, Providence.

ANNIE B. SHEPLEY. (American.)

230. S. Rosamond.

Loaned by ANNIE B. SHEPLEY.

WM. THORNE. (American.)

23I. Mrs. John Lawrence.

Loaned by WM. THORNE.

232. John Lawrence.

Loaned by WM. THORNE.

233. Mrs. Clarke.

Loaned by WM. THORNE 
AUGUSTUS VINCENT TACK. (American.)

234. Russell Sage.

Loaned by RUSSELI, SAGE RAPHAEL.

PRINCE TROUBETZKOY. (Russian.)

235. Portrait of Count S.

Loaned by P. P. TROUBETZKOY.

236. Mrs. Stilson Hutchins.

Loaned by' R. R. T.

GEORGE BURROUGHS TORREY. (American.)

237. Mme. G. B. Torrey.

Loaned by G. B. TORREY.

238. Wm. Gould Brokaw.

Loaned by G. B. TORREY.

MISS MARY E. TILLINGHAST. (American.)

239. Memorial Portrait on Glass of Mrs.

Laura McD. Stallo.

Loaned by MRS. AI.EX. MICDONAI.D.

JACOPO TINTORETTO, I5I8-I594. (Italian.)

240. Venetian Senators (from the Riccardi Palace).

Loaned by' T. J. BLAKESLEE. 
UNKNOWN.

24I. Mrs. Siddons.

Loaned by MRS. OWEN WISTER.

\section{A. MULLER URY. (American.)}

242. Miss Emily Hoffman.

Loaned by A. MULLER URY.

FREDERICK P. VINTON. (American.)

243. Thos. G. Appleton.

Loaned by FREDERICK P. VINTON.

\section{SIR ANTHONY VAN DYCK, I599-I64I. (Dutch.)}

244. Portrait of a Man.

Loaned by T. J. BLAKESLEE.

245. Count d'Alligre.

Loaned by GEO. J. GOULD.

\section{HURBERT VOS. (American.)}

247. Mrs. Henry Hollester Pease.

Loaned by HY. HOLLESTER PEASE. 
JAN VAN BEERS. (French.)

248. Miss Elsie de Wolfe.

Loaned by MISS DE WOLFE.

JAN VAN RAVESTEYN, I572-1657. (Dutch.)

249. Portrait of a Lady.

Loaned by DURAND RUEL.

\section{J. ALDEN WEIR. (American.)}

250. Portrait of a Young Lady.

Loaned by J. ALDEN WEIR.

25I. Mother and Child.

Loaned by J. ALDEN WEIR.

252. Miss Pierson.

Loaned by' J. ALDEN WEIR.

J. McN. WhistleR. (American.)

253. The Artist.

Loaned ly S. P. AVERY.

254. The Woman in White.

I.oned by CHAS. WHITTEMORE. 
IRVING R. WILES. (Amcrican.)

255. Mr. and Mrs. L. M. Wiles.

Loaned by IRVING R. WILES.

256. Miss Gertrude Coles.

Loaned by IRVING R. WILES.

257. Mrs. Stephen H. Brown.

Loaned by STEPHEN H. BROWN.

MRS. HENRY WHITMAN. (American.)

258. Henry L. Higginson.

Loaned by HENRY L. HIGGINSON.

E. B. WARING. (American.)

259. Harford Willing Hare Powell.

Loaned by E. B. WARING.

260. Portrait.

Loaned by E. B. WARING.

EMILE WAUTERS. (Dutch.)

26I. Mrs. Oscar L. Richard.

Loaned by MRS. OSCAR L. RICHARD. 
THEODORE WORES. (American.)

262. Portrait of Mrs. O.

Loaned by THEODORE WORES.

263. Portrait of Miss L.

Loaned by THEODORE WORES.

FREDERICK B. WILLIAMS. (American.)

264. Portrait of Mrs. W.

Loaned by FREDERICK B. WILLIAMS.

\section{A. L. ZORN. (Swede.)}

265. Mrs. Potter Palmer.

Loaned by Mrs. POTTER PALMRE.

266. Susan White Hildreth.

Loaned by SUSAN W. HILDRETH.

267. The Cigarette Girl.

Loaned by ROBT. W. DE FOREST. 
The following portraits arrived too late to be catalogued in their proper order:

\section{F. A. BRIDGEMAN. (American.)}

268. Mrs. W. A. Stone.

Loaned by F. A. BRIDGEMAN.

269. Mrs. F. R. Dunlap.

Loaned by F. A. BRIDGEMAN

W. H. FUNKE. (German.)

270. “Major” Sproull.

Loaned by W. H. FUNKE.

ALBERT LYNCH. (American.)

27I. Mrs. Gilbert E. Jones.

\section{LARGILLIÈRE. (French.)}

272. Lady as a Mythological Personage.

Loaned by STANFORD WHITE. 
GEORGE ROMNEY. (English.)

273. Sir John Reade, Bart.

Loaned by WM. L. ELKINS

AUGUSTUS ST. GAUDENS. (American.)

274. Robert Louis Stevenson (bas-relief).

275. Mildred and William Dean Howells (bas-relief). 


\title{
MINIATURES.
}

\author{
FRANK A. AIKEN.
}

30I. Mrs. H. G. P. and Child.

Loaned by FRANK A. AIKEN, EsQ.

302. Portrait of a Lady.

Loaned by FRANK A. AIKEN, EsQ.

\section{GREGORIA DE AJURIA.}

303. Miss Helen Brice.

Loaned by Senator CALVIN BRICE.

304. Portrait.

Loaned by Senator CALVIN BRICE.

305. Mrs. David Hennen Morris.

Loaned by MME. GREGORIA DE AJURIA. 306. Child of Mrs. Henry Hoyt.

Loaned by MRs. HENRY HOYT.

307. Mr. Charles Meyer.

Loaned by Mrs. CHARLES MEYER. 48 
308. Mlle. Koline.

Loaned by MME. GREGORIA DE AJURIA.

309. Children of Mrs. Chauncey Keep.

Loaned by MrS. CHAUNCEY KEEP.

\section{FISHER AMES.}

3Io. Mrs. Abausen Jermain.

Loaned by Mrs. RUSSELL SAGE.

3II. Major John Jermain.

Loaned by MRs. RUSSELL SAGE.

\section{WILLIAM J. BAER.}

312. Mrs. Henry W. Poor.

Loaned hy HENRY W. POOR, Es?

3I3. Mrs. Henry Fairchild James.

Loaned by MIss EDITH TRUE

314. Dorothea.

Loaned by MRS. BALLARD SMITH.

315. Charles E. Bushnell, Esq.

316. Mother and Child.

Loaned by EDW. R. HEWITT. 
3I7. Master Pembroke Jones.

Loaned by MrS. PEMBROKE JONES.

318. Mrs. J. Harry Wheeler.

Loaned by J. HARRY WHEELER, EsQ.

319. Sara.

Loaned by WILLIAM J. BAER, EsQ.

320. Miss $\mathrm{M}$.

Loaned by WILLIAM J. BAER, EsQ.

321. Miss Mildred Bliss.

Loaned by Mrs. WM. H. BLISS.

322. Miss Marian Litchfield.

Loaned by EDW. H. LITCHFIELD, EsQ.

\section{HELEN JOSEPHINE BAKER.}

323. Portrait.

Loaned by MISS HELEN JOSEPHINE BAKER.

324. Portrait.

Loaned by MISS HELEN JOSEPHINE BAKER.

325. Portrait.

Loaned by MISS HELEN JOSEPHINE BAKER.

326. Portrait.

Loaned by MISS HELEN JOSEPHINE BAKER. 
MARTHA IV. BAXTER.

327. Children of Mr. and Mrs. A. Lanfear Norrie.

Loaned by A. LANFEAR NORRIE, EsQ.

328. Mrs. Russell Sage.

Loaned by RUSSELL SAGE, EsQ.

329. Miss Wilder.

Loaned by WM. R. WILDER, EsQ.

330. Child of Mr. and Mrs. Arthur Leonard.

Loaned by ARTHUR G. LEONARD, EsR.

331. Mr. William Hoyt.

Loaned by Mrs. WM. HOYT.

332. Sister of Rev. John Wesley Brown, D.D.

Loaned by REv. JOHN W. BROWN, D.D.

333. Late Mrs. Baxter, widow of General Henry H. Baxter.

Loaned by MRS. ARTIIUR F. REES.

334. Miss Tower.

Loaned by LAWRENCE PHELPS TOWER, Es? .

M. LESLEY BUSH-BROWN.

335. Mr. J. Abner Harper.

Lraned by Mrs, M. L.ESLEY BUSII-BROWN. 
336. Lydia; a portrait.

Loaned by MRs. M. LESLEY BUSH-BROWN.

337. Dorothea.

Loaned by Mrs. HASTINGS HUGHES.

338. My Color Bearer.

Loaned by Mrs. M. LESLEY BUSH-BROWN.

339. Mr. Wm. Ricketts.

Loaned by MRS. R. BRUCE RICKETS.

\section{GEORGINE CAMPBELL.}

340. Mildred Dennis.

Loaned by MRS. WARREN E. DENNIS.

34I. Miss Meeker.

Loaned by MRS. MEEKER.

342. Portrait.

Loaned by Mrs. W. I). T.

343. Mrs. Billington.

Loaned by Mrs. BILLINGTON.

344. Portrait.

345. Mrs. S. M. Stevenson.

Loaned by MRs. STEVENSON.

346. Alfred Barham North.

Loaned by F. H. NORTH, EsQ. 


\section{GERTRUDE COLLES.}

347. Mrs. Louis M. Starr.

Louned by LOUIS M. STARR, Es?.

348. Miss Eleanor Greene.

Loaned by Major-General GREENE.

349. Miss Henrietta Danforth.

Loaned by MRs. EMALINE Y. DANFORTH.

350. Mrs. Arthur W. Little.

Loaned by Miss MARGARET WINSLOW

351. Miss Nella Wilder.

Loaned ly MRS. ENOS WILDER.

352. Mrs. E. F. Darrell.

Loaned by E. F. DARRELL, EsQ.

\section{MARIE CHAMPNEY.}

353. Mrs. H. as a "Geisha Girl."

Loaned by MISS MARIE CIAMMPEY.

354. Portrait Study.

Loaned by MISS MARIE CHAMPNEY.

355. Portrait of Miss C.

Loaned by MISS MARIE CHAMPNEY. 
356. Copy of Rubens' portrait of Baron de Vicq, in the Louvre.

Loaned by MIss MARIE CHAMPNEY.

357. Portrait of Master Frederick Strong, Jr.

Loaned by MISS MARIE CHAMPNEY.

358. Portrait Study of a Cardinal.

Loaned by Miss MARIE CHAMPNEY.

\section{MLLE. DEBREVAL.}

359. S. V. S. Wilder (1819).

Loaned by Mrs. CHARLES LEDYARD NORTON.

360. Mrs. S. V. S. Wilder (18I9).

Loaned by MRs. CHARLES LEDYARD NORTON.

36I. Mrs. Joseph Barrel (I8 I9).

Loaned by Mrs. CHARLES LEDYARD NORTON.

\section{DAVID.}

362. Mme. Sallé; a Dancer of the First Empire.

Loaned by ALEXANDER BLUMENSTEIL, EsQ.

MARY FRANCES ELMER.

363. Portrait of Mrs. Charles Morrogh.

Loaned by Miss MARY FRANCES ELMER. 
364. Portrait of Mr. H. Y. S. Smith.

Loaned by MISS MARY FRANCES ELMER.

36j. Portrait of Mr. Henry G. Marquand.

Loaned $b y$ Miss MARY FRANCES ELMER.

\section{. LYDIA FIELD EMMET.}

366. Portrait of a Baby.

Loaned by EVELENA D. STEVENS.

367. Anna Helena Emmet.

Loaned by MRS. T. L. EMMET.

368. Children of Robt. J. Hare Powel.

Loaned by ELIZABETH C. POWEL.

369. John U. A. Griswold.

Loaned by GEO. GRISWOLD, 2D.

370. Portrait of a Gentleman.

Loaned by Mrs. JOHN S. ELI.IS.

37 I. Portrait of a Lady.

Loaned by Mrs. JOHN S. ELLIS.

\section{EDWARD FESSER.}

372. Bishop Potter.

Loaned by EDWARD FESSER. 
373. Mrs. Geo. Edw. Wood.

Loaned by Mr. G. E. WOOD.

374. Mrs. Charles Goodhue.

Loaned by Mrs. PERCY R. KING.

375. Mrs. Henry Gillig.

Loaned by Mrs. HENRY GILLIG.

376. Mrs. Charles Kingdon.

Loaned by Mrs. GEO. JAY GOULD.

377. Mrs. E. Livingston Glover.

Loaned by Mrs. GLOVER.

378. Miss Edna Glover.

Loaned lby MRs. GLOVER.

379. Mrs. Geo. Howard Davison.

Loaned by G. H. DAVISON, EsQ.

380. Mrs. William Cross.

Loaned by B. FRANKLYN CROSS, EsQ.

381. Mrs. Rodewald.

Loaned by MISS LENA RODEWALD.

382. Mrs. Lucy Carroll.

Loaned by Mrs. CARROLL. 


\section{LUCIA FAIRCHILD FULLER.}

383. Mary and Katherine Amory.

Loaned by MrS. HENRY B. FULLER

384. Portrait of E. T. H. Talmage, Jr.

Loaned by Mrs. HENRY B. FULLER,

385. Portrait of Miss Jina Hardy.

Loaned by Mrs. HENRY B. FULLER.

386. Portraits of Florence and Margaret La Farge.

Loaned by Mrs. C. GRANT LA FARGE.

387. Portrait of Miss Constance Thill.

Loaned by Mrs. HENRY B. FULLER.

388. Portrait of Charles P. Curtis, 3d.

Loaned by MRS. HENRY B. FULLER.

$$
\text { J. GAERIN. }
$$

389. Marie Antoinette.

Loaned by JULIA CHESTER IVELIES.

$$
\text { ALICE HAM. }
$$

390. Portrait of Mrs. C.

Loancd by MRS. CONGOR. 
39I. Portrait of Mrs. M.

Loaned by Miss ALICE HAM.

392. Portrait of Miss $\mathrm{H}$.

Loaned by LAYTON BREWER, EsQ.

393. Portrait of Miss H.

Loaned by CHAS. H. HAM, EsQ.

\section{LAURA C. HILLS.}

394. Case of Miniatures.

Loaned by MIss LAURA C. HILLS.

\section{JOHN HOPPNER.}

395. Miss Bover.

Loaned by Miss JULIA CHESTER WELLES.

\section{GERTRUDE HURLBUT.}

396. Commodore Postley.

Loaned by Mrs. CLARENCE POSTLEY.

397. Mrs. Brinsmade.

Loaned by MRs. BRINSMADE.

398. Lady Beatrice Butler.

Loaned by Mrs. GERTRUDE HURLBUT. 
399. Duchess of Marlborough.

Loaned by MKS GIRTRUDE HURLBUT.

400. Miss H. G. Longstreet.

Loaned by MISS LONGSTREET.

40I. Miss Egan.

Loaned by MIss EGAN.

402. Master George Kirkland.

Loaned by Mrs. KIRKLANI).

403. Mrs. C.

404. Mrs. Kingdon.

Loaned by Mrs. GEO. J. GOULD.

\section{JERVIS.}

405. Albert Gallatin.

Loaned by Mrs. GRENVILLE KANE.

406. Mrs. Henry Breevort.

Loaned by Mrs. GRENVILLE KANE.

407. Baron Charles Auguste de Gironcourt.

Loaned $b^{\prime}$ Mrs. GRENVILLE KANE.

\section{ISAAC A. JOSEPHI.}

408. Mrs. C. W. Carpenter.

Loaned by MRS. CARPENTER. 
409. Miss M. T.

Loaned by ISAAC A. JOSEPHI, EsQ.

4 Io. Mrs. Dikran Kelikian.

Loaned by DIKRAN KELIKIAN, Eso.

4 I . Miss J. T.

Loaned by ISAAC A. JOSEPHI, ESQ.

412. Mrs. F. St. M. Stewart.

Loaned by MRs. STEWART.

4I3. Mrs. E. Van Zandt Wheeler.

Loaned by MRs. WHEELER.

4I4. T. R.

Loaned by' ISAAC A. JOSEPII, EsQ.

\section{ROBERT KEELING.}

4I 5. Mrs. W. C. Whitney.

4I6. Miss Alice Belknap.

417. Miss Sylvia Meyers.

4I8. Mr. Joseph Leidy.

4I9. Mrs. Ben Tilghman. 


\section{SHIRLEY TURNER.}

420. Boy.

Loaned by MISS SHIRLEY TURNER.

42 I. Girl.

Loaned by MISS SHIRLEY TURNER.

\section{JULES LAURE.}

422. Portrait of John P. Barre.

Loaned by LEON BARRE, Eş2.

423. Portrait of Mrs. John P. Barre.

Loaned by' LEON BARRE, Es?.

\section{MARGARET M. LUTHER.}

424. Gen. Thomas A. Davis.

Loaned by GEN. THOS. A. DAVIS.

425. Portrait.

Loaned by MRS. GEO. LEWIS.

\section{MALBONE.}

426. Mrs. Amory, of Boston.

Loaned by MISS HELEN AMORY ERNST. 
427. Mrs. John Chipman Gray.

428. Miss Louisa P. Loring.

429. Mrs. Gustave Lürman.

430. Mrs. Rockwood Hoar.

43I. Mrs. Henry Oothout.

\section{MICHAELS.}

432. Portrait.

Loaned by Mrs. PIERRE LORILLARD.

\section{PIERRE MIGNARD.}

433. Mme. de Fontanes, Maid of Honor at the Court of Louis XIV.

Loaned by ALEXANDER BLUMENSTEIL, EsQ

$$
\text { J. OTIS MINOT. }
$$

434. Miss Helen Ashton Bloodgood.

Loaned by MRS. WILLIAM BLOODGOOD.

435. Mrs. Edgerton L. Winthrop, Jr.

Loaned by J. OTIS MINOT, EsQ

436. Miss Lillie Gardner.

Loaned by J. OTIS MINOT, EsQ. 
MRS. MORGAN.

437. Portrait of a Lady.

Loaned by MRS. CHAS. DE RHAM.

438. Portrait of Two Children.

Loaned by MRS. CHAS. DE RHAM.

\section{RHODA HOLMES NICHOLLS.}

439. Mrs. Alice Trenholm.

Loaned by Mrs. TRENHOI.M.

440. Miss Rittenhouse.

Loaned by MRS. NICHOLLS.

44I. Mrs. Carleton Cootes.

Loaned by MRs. COOTES.

\section{LILLIE V. O'RYAN.}

442. Portrait of Mr. Gordon L. Ford.

Loaned by MRS. ROSIVELL SKEEL, JR.

443. Children of Mrs. William Barr.

Loaned by MRS. WILIIAM BAKR.

\section{R. CURZON POULTNEY.}

444. Miss Madeline Ives Goddard.

Loaned by MRS. R. H. I. GODDARD. 


\section{4 JOHN ROBINSON.}

445. Benjamin West.

Loaned by MRs. JAWOOD LUKENS.

\section{ROGERS.}

446. Mrs. Margaret Presian Jermain Slocum.

Loaned by Mrs. RUSSELL SAGE.

447. Mr. A. Jermain.

Loaned by Mrs. RUSSELL SAGE.

\section{J. STAPLES ROWE. (Cornish.)}

448. The late Mr. Jay Gould and Wife.

Loaned by Miss HELEN GOULD.

449. Theodore Havemeyer, Esq.

Loaned by J. STAPLES ROWE, EsQ.

450. Jesse Seligman, Esq.

Loaned by Mrs. WASSERMAN.

45 I. Mrs. Jerome Hurlburt.

Loaned by MRs. ALFRED RAY.

452. Mrs. Edward H. Litchfield.

Loaned by EDWARD H. LITCHFIELD, EsQ. 
453. Miss Litchfield.

Loaned by EDWARD H. LITCHFIELD, EsQ.

454. Portrait of Miss M.

Loaned by J. STAPLES ROIVE, EsQ.

455. Mrs. L. M. Hirsch.

Loaned by J. STAPLES ROWE, EsQ.

456. Martha and John Hicks.

Loaned by MISS T. F. HICKS.

\section{GEORGE SHUMMAY.}

457. Old Gentleman.

Loaned by MIsS SARAH II. HOWE.

458. Old Lady.

Loaned by MISS SARAH M. HOWE.

\section{ANN SHAW.}

459. Mrs. Hull.

Loaned by MIss A. L. SHAW.

\section{GEORGE SLOANE.}

460. Portrait of C. A. I.

Loaned by Mrs. AUGUSTINE JONES. 


\section{H. VANCE SWOPE.}

461. Zelia Farges.

462. Mrs. De Heredia and Miss S.

463. Mrs. A. C. Farges.

- 464. Pierre Farges.

\section{EMILY DAYTON TAYLOR.}

465. Mrs. Charles M. Lea.

Loaned by MRs. J. MADISON TAYLOR.

466. Miss Edith Moore Taylor.

Loaned by MRS. J. MADISON TAYLOR.

467. Miss Marian Taylor.

Loaned by MRs. J. MADISON TAYLOR.

JOHN CAMPBELL TELFER (see also page 88). 468. Mrs. Mercer.

Loaned by MRS. J. R. MERCER.

469. Miss Waters.

Loaned by' J. C. TELFER, EsQ.

470. Ṃiss Ellliot, 


\section{ELKANAH TISDALE.}

471. Mr. and Mrs. T. Green Pierson (1812).

Loaned by MRs. GEORGE W. MURDOCK.

\section{MARY C. TRASK.}

472. Portrait of Mrs. J. D. T.

Loaned by MRS. J. D. TRASK.

LUCY PARKMAN TROWBRIDGE.

473. Mrs. James Roosevelt.

Loaned by MRS. JAMES ROOSEVELT.

474. Mrs. Dawson Coleman.

Loaned by MRS. BURNEY.

475. Master Herman Livingston Rogers.

Loaned by ARCHIBOLD ROGERS, EsQ.

476. Miss Helen Southgate Hotchkiss.

Loaned by MISS LUCY PARKMAN TROWBRIDGE.

477. Master Henry Roger Benjamin.

Loaned by WM. E. BENJAMIN, EsQ.

478. Mrs. Wm. Evartt Benjamin.

Loaned by WM. E. BENJAMIN, Es?.

479. Miss Trowbridge.

Loaned by MISS LUCY PARKMAN TROWBRIDGE. 
480. Mr. H. Howard Hart.

Loaned by MRS. HENRY BURDEN.

481. Miss Myrick.

Loaned by MIss LUCY PARKMAN TROIVBRIDGE.

482. Professor Dana.

Loaned by MRs. DANA.

\section{MRS. WASHBURN.}

483. Mrs. Mason F. Cogswell.

Loaned by MRS. CHARLES LEDYARD NORTON.

\section{C. \& F. WEIDNER.}

484. Boy.

Loaned by CHAS. WEIDNER, EsQ.

485. Girl.

Loaned by CHAS. WEIDNER, EsQ.

486. Mrs. Levi P. Morton.

Loaned by MR. LEVI P. MORTON.

487. Mrs. Hamilton W. Cary.

Loaned by MRS. BOSTWICK.

488. Mrs. W. Watts Sherman.

Loaned by MRs. W. WATTS SHERMAN. 
489. Portrait.

Loaned by CHAS. WEIDNER, EsQ.

490. Mrs. Morris Herrmann.

Loaned by MRS. HERRMANN.

49I. Mrs. Castairs.

Loaned by MRS. BOSTIVICK.

492. Miss Milly Lowery.

Loaned by MISS LOWERY.

493. Mrs. W. Watts Sherman.

Loaned by MRS. W. WATTS SHERMAN.

494. The Misses Sherman.

Loaned by MRs. W. IVATTS SHERMAN.

495. Mr. George R. Schieffelin.

Loaned by MRS. GEORGE R. SCHIEFFELIN.

\section{EDITH DEAN WEIR.}

496. Portrait.

Loaned by MISS W'EIR.

WM. J. WHITTEMORE.

497. Mrs. Charles Whittemore.

Loaned by WM. J. WHITTEMORE, EsQ. 
498. Mrs. W.

Loaned by WM. J. WHITTEMORE, EsQ.

499. Mrs. B.

Loaned by GEO. H. BOGERT, EsQ.

\section{UNKNOWN.}

500. Empress Josephine.

Loaned by HENRY WATROUS, Esq.

501. Portrait of Mlle. Fayolle.

Loaned by LÉON BARRE, EsQ.

502. Mrs. Henry Gilbert Livingston.

Loaned by MISS ALICE LIVINGSTON ELDRIDGE.

503. Nathaniel Richards (I 825 ).

Loaned by MRS. CHARLES LEDYARD NORTON.

504. Mason F. Cogswell (I 820).

Loaned by Mrs. CHARLES LEDYARD NORTON.

505. Catherine de' Medici.

Loaned by Miss JULiA CHESTER WELles.

506. Katherine of Russia.

Loaned by Miss JULIA CHESTER WELLES.

507. An English Beauty of 1760 .

Loaned by Miss JULIA CHESTER WELLES. 
508. A French Box Miniature.

Loaned by Miss JULIA CHESTER WELLES.

509. Louisa Matilda of Bayrout.

Loaned by Miss JULIA CHESTER WELLES.

5io. A Court Lady.

Loaned by Miss JULIA CHESTER WELles.

5I I. Duchess of Beni.

Loaned by Miss JULIA CHESTER WELLES.

5I2. Mrs. Bethune.

Loaned by Mr. ROBERT MCCARTER.

\section{RICHARD COSWAY.}

5I3. Duchess of Devonshire.

Loaned by J. W. BOUTON, EsQ.

\section{MANZONI.}

514. Lady Vansittart.

Loaned by J. W. BOUTON, Esก̨.

515. Princess of Wurtemburg.

Loaned by J. W. BOUTON, Ese.

GENARE.

516. Lady Playing Harp.

Loaned by J. W. BOUTON, EsQ. 
PLIMER, PUPIL OF COSWAY.

5I7. Portrait of Three Young Ladies.

ANNIE M. HILLS.

518. Miss Mary Isabel Collins.

Loaned by MRS. R. MCCLELLAN COLLINS.

519. Mrs. Gary (wife of former Postmaster Gary).

Loaned by JAS. A. GARY, EsQ.

\section{J. STAPLES ROWE.}

520. Mrs. M. A. Tyler.

Loaned by MRs. M. A. TYLER.

\section{MARTHA S. BAKER.}

52I. Portrait.

Loaned by MARTHA S. BAKER.

522. Mrs. John R. Key.

Loaned by MARTHA S. BAKER.

523. Little Miss Giffin.

Loaned by MARTHA S. BAKER.

524. Mother and Child.

Loaned by MARTHA S. BAKER.

525. Miss Olga Oldberg.

Loaned by MARTHA S. BAKER.

526. Miss Ellen Walch.

Loaned by MARTHA S. BAKER.

527. Master Virgil Oldberg.

Loaned by MARTHA S. BAKER. 


\section{COLLECTION OF 237 MINIATURES \\ LENT BY PETER MARIÉ, ESQ.}

528. Mrs. Cleveland, wife of the ARTIST. ex-President. F. Paillet.

529. Mrs. Morton, wife of the ex-

Vice-President.

Carl A. Weidner.

530. Mrs. John Lee Carroll

(Carter Thompson), wife of the ex-Governor of Maryland.

F. Paillet.

531. Mrs. Jerome Bonaparte (Caroline Appleton), granddaughter of Daniel Webster.

532. Mrs. Wetmore (Keteltas), wife of the U.S. Senator.

533. Mrs. David Lydig (Hannah Tompkins).

534. Mrs. Royal Phelps Carroll (Langdon).

Mrs. Behenna.

535. Mrs. W. Watts Sherman (Carter Brown). Carl A. Weidner.

536. Mrs. J. Lawrence Lee (Margaret Livingston). F. Paillet.

537. Comtesse Harold de Moltke-

Hvitfeldt (Anna Hutton). Carl A. Weidner. 
538. Mrs. William Douglas Sloane (Emily Vanderbilt). F. Paillet.

539. Mrs. William Waldorf Astor (Paul).

540. Mrs. Samuel S. Howland (Belmont).

Carl A. Weidner.

54I. Mrs. Judge John Clinton Gray (Grace Townsend). 66

542. Mrs. Robert B. Minturn (Shaw).

F. Paillet:

543. Mrs. Hy. Dwight Sedgwick,

Jr. (Sara May Minturn).

544. Mrs. Isaac Newton Stokes

(Edith Minturn).

545. Mrs. Charles A. Post (Marie

C. de Trobriand).

546. Mrs. Benjamin Welles

(Frances Swan).

547. Mrs. J. Kennedy Tod

(Maria Howard Potter).

548. Mrs. Wm. Butler Duncan (Sargent).

549. Mrs. Wilton Phipps (Jessie Duncan).

550. Mrs. Edward Wharton

(Edith Jones).

55 I. Mrs. Frances Amory (Grace Minot). 
552. Mrs. Roland Redmond (Helen Bulkley).

553. Mrs. Campbell P. Steward (Margaret A. Beekman).

554. Mrs. Gerald Hoyt (Appleton).

555. Mrs. Henry W. Livingston (Mary S. McRa).

556. Mrs. Bache Emmet (Anna Monson).

557. Mrs. Neilson (daughter of the late Admiral Rodgers).

558. Mrs. Stanley Mortimer(Elizabeth Livingston Hall).

559. Mrs. Charles H. Marshall (Josephine Lenox Banks). 560. Mrs. H. Van Rensselaer Kennedy (Marian Robbins).

56r. Countess of Strafford (Cora Smith Colgate).

562. Mrs. Karrick Riggs (Pauline Oothout).

563. Mrs. Edwin D. Morgan (Elizabeth Moran).

564. Mrs. Senator Don Cameron (Sherman). 
565. Miss Edith Wetmore.

F. Paillet.

566. Mrs. James Abercrombie Burden (Mary Irvin).

567. Mrs. John C. Wilmerding (Georgiana Heckscher).

568. Mrs. Griswold Gray (Susan Irvin).

569. The Marquise de Mores (Medora von Hoffmann).

570. Mrs. Victor Drummond (Lily Lamson), wife of the British Minister at $\mathrm{Mu}$ nich.

57I. Mrs. Colonel William Goddard, of Providence R. I.

572. Mrs. Adolph Ladenburg (Emily Stevens).

573. Mrs. Clement C. Moore.

574. Mrs. Duncan Elliott (Sallie Hargous).

575. Mrs. Charles A. Sackett (Mary Alice Townsend).

576. Mrs. Buchanan Winthrop (Sallie Townsend).

577. Mrs. Archibald Hopkins and Mrs. J. W. Miller(daughters of Gov. Wise of Virginia and granddaughters of Edward Everett). 
578. Mrs. John Steward, Jr.
(Cornelia S. Jones).
F. Paillet.

579. Mrs. John Hobart Warren.

580. Mrs. Sidney Dillon Ripley.

581. Mrs. Henry Asher Robbins

(Elizabeth Bend).

582. Mrs. Sackville-West.

583. Mrs. Francis McNeill Bacon, Jr. (Pauline Post).

584. Mrs. Lawrence Turnure, Jr.

(Romaine Stone).

585. Mrs. Edwards Spencer

(Stout-Emily Meredith Read).

586. Mrs. Wm. Jay Schieffelin

(Marie Louisa Shepard) (after picture by B. C. Porter).

587. Mrs. Horatio Odo Cross

(Florence Griswold).

588. Mrs. C. M. Wright (Caroline May).

589. Miss Amy Bend.

590. Mrs. Stewart (Susan Sturgis).

591. Mrs. Scott (Mary Sturgis).

592. Lady Curzon (Mary Leiter, wife of the Viceroy of India).

593. Miss Grau Howard Potter. 
594. Miss Bertha Howard Potter.

F. Paillet.

595. Mrs. William P. Jaffray

(Helen Smythe).

596. Mrs. C. Oliver Iselin (Hope

Goddard).

597. Miss Elizabeth McClelland

Greene.

598. Miss Mignonne Woodworth.

599. Miss Susan Whittier.

6oo. Miss Margaret Clarkson.

6oI. Mrs. Robert Le Roy (Lewis).

602. Mrs. Arthur Henry Paget

(Mary Fiske Stevens).

603. Mrs. Jordan L. Mott, Jr.

604. Mrs. W. Storrs Wells.

605. Lady Terence T. Blackwood

(Flora Davis).

606. Mrs. Richard McCreery

(Edith Lorillard Kip).

607. Mrs. Frederick Edey (Sarah Birdsall Otis).

608. Mrs. Charles de Kay (Edwalyn Coffee).

609. Mrs. Auguste P. Montant

(Hannah Townsend).

6ı. Mrs. Hy. Pennington Tailer

(Cora Wright). 
6iı. Mrs. Richard Irvin (Mary Morris).

6r2. The Misses Rives (now Mrs. Walker Breese Smith, and Mrs. John Borland).

6r 3. Mrs. Boissevain (Magee).

614. Miss Helen Horton.

615. Miss Marie Reed.

6ı6. Mrs. Henry J. Hoffman (Bertha Whelan).

6r7. Mrs. Leslie Cotton (Mary Clarkson Potter).

6i8. Mrs. Iselin (Garner).

6ig. Mrs. Henry S. Crane (Charlotte Winthrop).

620. Mrs. Elliott Roosevelt (Anna Hall).

621. Miss Handy.

622. Mrs. David Lydig (Hannah Tompkins).

623. Mrs. Lloyd Bryce (Edith Cooper).

Mrs. Behenna.

624. Mrs. Gambrill (Anna Van Nest).

625. Mrs. Benjamin C. Porter.

626. The Lady Playfair (Edith Russell). 
627. Mrs. Henry Vincent Hig-

gins (Mary L. Parsons). Mrs. Behenna.

628. Mrs. Wm. H. L. Lee (McLane).

ARTIST:

629. Mrs. Frederick Sheldon (Fearing).

630. Princess Pierre Troubetskoy (Amèlie Rives).

63I. Hon. Mrs. Dudley Leigh (Helen Beckwith).

632. Madame de Lopez-Roberts (Angela Terry).

633. Mrs. Frederic Grand d'Hauteville (Susan McComb).

634. Baronne de Charrette (Antoinette Polk).

635. Miss Ethel Cram.

636. Mrs. T. Woodward Haven (Henriette Cram).

637. Mrs. Richard Howland Hunt (Carley).

638. Mrs. Victor Sorchan (Charlotte Hunnewell).

639. Miss Choate.

640. Miss Perkins.

641. Miss Margaret AuchmutyMackay.

642. Miss Mary Pearsall Field. 
643. Mrs. H. Casimir de Rham (Georgiana Berryman).

644. Mrs. Frederic Tams (Blanche Spedden).

645. Mrs. Frederic Gebhard (Louise Morris).

646. Mrs. Sidney Smith (Fanny Tailer).

647. Miss Lydia Emmet.

648. Miss Jane Emmet.

649. Miss Mabel Van Rensselaer.

650. Miss Cora Randolph.

65 I. Mrs. George Griswold (Post).

652. Mrs. William P. Jaffray

(Helen Smythe).

653. Mrs. Clarence Cary (Elizabeth M. Potter).

654. Countess of Essex (Adele Grant).

655. Mrs. Ronalds (Mary Frances Carter).

656. Mrs. Wm. Bayard Cutting (Olivia Murray).

657. Mrs. Edmund L. Baylies (Van Rensselaer).

658. Mrs. J. P. Morgan, Jr., (Jane N. Grew). 
659. Mrs. Alexander Brown, of

Artist.

\section{Baltimore.}

660. Miss Louise Dexter.

66r. Mrs. Francis Vinton Hoppin (Sarah Weekes).

662. Mrs. Fenno (Thorndike).

663. Mrs. Burke Roche (Fanny Work).
Mrs. Behenna.

“

66

66

664. Miss Laura Jenkins, of Baltimore.

665. Mrs. John B. Thayer, of Philadelphia.

666. Niss Beatrice Bend.

667. Miss Janet Hoyt.

668. Miss Ada Godfrey.

669. Mrs. Wm. Post (Anthony).

670. Miss Rita Carson, of Philadelphia.

Carl A. Weidner. 
677. Mrs. Richard Stevens, Jr., of

ARTIST.

Castle Point, Hoboken. Carl A. Weidner.

678. Mrs. John G. Heckscher

(Mary Travers).

679. Mrs. Augusta Temple Merritt (Schack).

680. Miss Bessie Frelinghuysen Davis.

68 I. Miss Lucy Byrd.

682. Mrs. Robert J. Hare Powel and her Children (Elizabeth Crosby).

683. Miss Betsy Riddle, of Philadelphia.

684. The four daughters of $\mathrm{Mr}$.

Heber R. Bishop. viz. :

Mrs. J. Low Harriman,

Mrs. James Lanier,

Mrs. Moses Taylor,

Miss Bishop.

685. Mrs. Ellis Hoffman (Sybil Sherman).

686. Miss Helen Van Cortlandt Morris.

687. Mrs. H. de Berkeley Parsons (Frances Walker).

688. Mrs. F. Egerton Webb (May Randolph). 
689. Miss Angelica Schuyler Crosby.

Carl A. Weidner.

690. Mrs. F. S. Watson (Mary

Perkins), of Boston.

69r. Miss Mary Wolfe Hoffman.

692. Miss Dorothea Wolfe Hoffman.

693. Mrs. Rathbone Bacon (Vanderbilt Barker).

694. Mrs. Harold H. Baring (Marie Churchill).

695. Miss Carolina Washington Bond (great-niece of George Washington).

696. Mrs. Frederic Gebhard (Louise Morris).

Edward Fessner.

697. Miss Morris, of Baltimore (in Reveries of a Bachelor at Bar Harbor).

Edward Tessa.

698. Mrs. Ralph Miller (Alice Bowers Lee).

Carl A. Weidner.

699. Miss Marion Bowers Lee.

700. Mrs. Wm. Allen (Anderson).

701. Miss Daisy Post.

702. Mrs. T. J. Oakley Rhinelander (Edith Cruger Sands). 
703. Mrs. J. Lee Tailer (Marie Stirling).

ARTIST.

Carl A. Weidner.

704. Miss Mabel Burke of Llewellyn Park.

Edward Fesser.

705. Mrs. Wm. B. Dinsmore,

Jr. (Marion de Peyster Carey).

706. Mrs. Charles Stuart Dodge (Flora Bigelow).

Galvini.

707. Mrs. Isaac Bell (Mott).

Mrs. Behenna.

708. Mrs. Van Rensselaer Cruger (Julie Storrow).

F. Paillet.

709. Mrs. Eugene Van Rensselaer (Sarah Pendleton). Mrs. Behenna.

7 10. Princess Brancaccio (di Triggiano).

7II. Mrs. Burke Roche (Fanny Work).

7I 2. Mrs. Potter Palmer.

Paillet.

Mrs. Behenna.

713. Mrs. Julian Story (Emma Eames).

714. Mrs. Francklyn (Miss Hoyt).

715. Mrs. Henry Clews.

Paillet.

Mrs. Behenna.

716. Mrs. Lucius Wilmerding (Caroline Murray).

717. Lady Frenkland (di Zerega). 718. Mrs. Daniel Fearing(Strong). Paillet. 
7ig. Mrs. Herman Emmett (Johnson).

ARTIST.

Mrs. Behenna.

720. Mrs. Egerton L. Winthrop,

Jr. (Hecksher).

Paillet.

72 I. Mrs. Dominguez (Murphy). Mrs. Behenna.

722. Mrs. Hamilton Fish Webster (Post).

723. Miss Amy Townsend.

724. Miss Ogden.

Paillet.

725. Miss Emily Vanderbilt

Sloane.

726. Miss Brewster.

727. Miss Marie Winthrop.

728. Miss Edith Knowlton.

Mrs. Behenna.

Paillet.

Carl Weidner. Mrs. Behenna.

Paillet.

Mrs. Behenna.

729. Mr. Marié's Great Grandmother, painted about I770.

730. Mr. John B. Marie, father of the exhibitor, painted I8I8.

731. Mrs. John B. Marié, mother of same, painted I8I8.

732. Mrs. John B. Marié, mother of same, in 1865.

733. Mr. Arnaud, grandfather of same, about 1790.

734. Mrs. Arnaud, grandmother of same.

735. Mrs. Sauer (Léontine Marié).

736. Mrs. F. K. Pendleton (Sallie Marié).

737. Mrs. F. K. Pendleton (Sallie Marié).

738. Mrs. José M. Munoz (Leontine Thièriot).

739. Madame Andréi (Henriette de Bermingham).

740. Mrs. Morgan Gibbs Barnwell (Lily Marié). 
ARTIST.

741. Mrs. Percy C. Madeira (Marié Marié).

742. Mrs. Fritz E. Suse (Léontine Sauer).

743. Miss Léontine Marié.

744. Miss Josephine Marié.

745. Mrs. Leon Marié (Carolyn Hoe).

746. Mrs. John B. Marié (Florence Waite).

747. Mrs. Louis Marié (Lee Duval).

748. A Young Lady (from Tiffany \& Co.).

749. Mrs. Tickell.

Isabey.

Cosway.

750. A Lady.

751. Miss Foote, Comedienne (afterwards Countes of Harrington).

752. Comtesse de $\mathrm{T} \longrightarrow$, about 1788.

753. Mademoiselle, Daughter of Henry IV and Marie de Medicis.

(From Migniac Sile, Lot 303. Londun, 18, .)

754. A Lady. (From Magniac Sale, Lot 320.)

755. Genrgina Spencer, Duchess of Devonshire (the famous partisan of Charles James Fox). 756. A Lady. 757. A Lady. 758. Laciy Curzon-enamelled on silver.

759. First Balloon Ascent at St. Cloud about 178 S. 760, A Neapolitan Boy, isth Century. 
Artist.

761. A Neapolitan Girl, I8th Century.

762. Mrs. Sidney Webster.

Weidner.

763. Mrs. F. K. Pendleton.

\section{MISS MILLSPAUGH.}

764. Portrait.

Loaned by Miss MILLSPAUGH.

\section{IDA M. WILDE.}

765. Mrs. Wm. H. Cooper.

Loaned by MRs. COOPER.

766. Mrs. Chas. McKinstry Nichols.

Loaned by Mrs. NICHOLS.

767. Mrs. Harold W. Wilde.

Loaned by MRs. WILDE.

768. Miss Mary Van Buren.

Loaned by MISS VAN BUREN.

769. Mrs. Oliver James Delamare.

Loaned by MRs. DELAMARE.

770. Mrs. Wm. Franklin Homan.

Loaned by MRS. HOMAN.

$$
\text { J. C. TELFER (see also p. 66). }
$$

771. Mrs. Telfer.

Loaned by J. C. TELFER.

772. Miss Julia Opp.

Loaned by J. C. TELFER.

773. Mr. Mercer.

Loaned by MRs. MERCER. 


\section{INDEX OF SUBJECTS.}

M. stands for Miniature.

Allen, Frederick Stevens, IOI.

Alexander, Charles B., 42.

Alexander, Mrs. Charles B., I84.

Alexander, Jas. W., 5.

Anderson, Mrs. S. L., II.

André, Major, I3.

Austin, Miss, 3.

Avery, Hon. B. Parke, 28.

Adams, Edward D., I32.

Amory, Mary and Katherine, M.

Amory, Mrs., of Boston, M.

Appleton, Thos. G., 243.

Antoinette, Marie, M.

Astor, Mrs. John Jacob, 525.

Bliss, Mrs. Wm. H., M.

Bliss, Miss Mildred, M.

Bushnell, Charles E., Esq., M.

Brown, Mrs. Stephen H., 257.

Barre, John P., M.

Barre, Mrs. John P., M.

Barr, Mrs. William, Children of, M. 
Belknap, Miss Alice, M.

Butler, Lady Beatrice, M.

Brinsmade, Mrs., M.

Benjamin, Master Henry Roger, M.

Benjamin, Mrs. Wm. Evartt, M.

Barrel, Mrs. Joseph (1819), M.

Baxter, Late Mrs., widow of General Henry H.

Baxter, M.

Beard, Wm. H., I 82.

Bliss, E. W., 163 .

Bonar, Mrs., and her Daughter Agnes, 208.

Boyd, General, 226.

Brice, Calvin S., 218.

B., Mrs., 7 .

Bjorksten, Mme., i I I.

Blue Boy, I I4.

Bathurst, Lady, 158.

Boy and Dog, I 24.

Baker, Geo. F., 86.

Beckwith, Mrs., 3 I.

Blackwood, Lady Terence, 83.

B., Mrs. M., I08.

Botticelli, 37 .

Bloodgood, Miss Helen Ashton, M.

Bethune, Mrs., M.

Billington, Mrs., M.

Bover, Miss, M.

Brevoort, Mrs. Henry, M.

C., Miss, M.

Coleman, Mrs. Dawson, M.

Cootes, Mrs. Carleton, M.

Carpenter, Mrs. C. W., M. 
Coles, Miss Gertrude, 256.

Cannon, Col., 29.

Cary, Mrs. Hamilton W., M.

Castairs, Mrs., M.

Cogswell, Mrs. Mason F. (IS20), M.

Colden, Governor, and his Grandson, Warren De Lancey, 68. .

Chanler, Miss, I 52.

Claypoole, Mrs., I 54.

Conkling, Mrs. A. R., 145.

C. H., Miss, I4I.

C., Mrs., I 42.

Coyte, Geo., Portrait of, I 16.

Crimmins, John D., 107.

Carroll, Mrs. Royal Phelps, I79.

Clarke, Mrs., 233.

Country, 175.

Curtis, Charles P., 3d., M.

Cross, Mrs. William, M.

Carroll, Mrs. Lucy, M.

Cooper, Mrs. Wm. H., M.

C., Mrs., M.

C., Mrs., M.

Collins, Miss Mary Isabel, 5 I S.

Devonshire, Duchess of, 513.

de Wolfe, Elsie, 248.

de Kay, Minna Craven, IO3.

de Laugier Villars, Countess, 52.

de R., Baroness Mathilde, I 34 .

de Villare, Duchess, I6 I.

de Fontanes, Mme., Maid of Honor at Court of Louis XIV., M. 
Dahlgren, Mrs. J. Vinton, 7I.

Dorothea and Francesca, 42.

Douglas, Miss Harriet, 26.

Danforth, Mrs. Emaline Y., M.

Darrell, Mrs. E. F., M.

Davison, Mrs. Geo. Howard, M.

Delamare, Mrs. Oliver James, M.

Dorothea, M.

Duer, Wm. A., President Columbia College, r6.

Dancing Girl, I23.

Dagnan-Bouveret, Mme., 87.

Davis, Gen. Thomas A., M.

Dana, Professor, M.

De Heredia, Mrs. and Miss S., M.

Dorothea, M.

Dennis, Mildred, M.

Duchess of Beni, M.

Dunlap, Mrs. F. R., 269.

Emmet, Anna Helena, M.

Edgerton, Mrs. Wright P., 45.

Ely, Mrs. Geo. B., 62.

E., Miss, M.

Egan, Miss, M.

Elliot, Miss, M.

French, Mrs. Henry F., 4.

Frelinghuysen, Mrs. Theodore, 40.

Fish, Hamilton, 43.

Fairfax, Lord, 147.

Francke, Mrs. Leopold H., I27.

Flagg, Mrs. W. Allston, 106.

Forest, Miss de, 222.

Farges, A. C., M. 
Farges, Mrs. Piere, M.

Farges, Zelia, M.

Fayolle, Mlle., M.

Ford, Mr. Gordon L., M.

Gray, Mrs. John Chipman, M.

Gladstone, Right Hon. Wm. E., 129.

Godwin, Parke, 178.

Graves, Mrs. C., I66.

Gill, Miss Catharine, 36.

Glaenzer, Mrs. Eugene, 55.

Goodridge, Miss Caroline L., 46.

Graham, John A., LL.D., 70.

Giffin, Miss, M.

Greene, Miss Eleanor, M.

Griswold, John U. A., M.

Goodhue, Mrs. Charles, M.

Gillig, Mrs. H., M.

Glover, Mrs. E. Livingston, M.

Glover, Miss Edna, M.

Gironcourt, Baron Charles Auguste de, M.

Gallatin, Albert, M.

Gary, Mrs., M.

Goddard, Miss Madeline Ives, M.

Gardner, Miss Lillie, M.

Gould, Jay, and Wife, M.

Gary, Mrs. (wife of former Postmaster Gary), 519.

Gilbert, Mrs. H. Bramhall, 524.

Giffin, Little Miss, M.

Hardy, Miss Jina, Portrait of, M.

Harper, Mr. J. Abner, M.

Hewitt, Hon. Abram S., I7.

Hoyt, Mr. William, M. 
Huntington, Miss Marie, I28.

Harrison, Dorothy, I 59.

H., Miss, Iog.

Hart, Mr. H. Howard, M.

Hull, Mrs., M.

Hoar, Mrs. Rockwood, M.

Hotchkiss, Miss Helen Southgate, M.

Higginson, Henry L., 258.

Hildreth, Susan White, 266.

Hoffman, Miss Emily, 242.

Hutchins, Mrs. Stilson, 236.

Hammond, Mrs., I73.

Hammond, Mr., 206.

Hoffman, Mrs. Francis Burrall, 2.

Herrmann, Mrs. Morris, M.

H. B., Mrs., I4.

Havemeyer, Theodore, M.

Hurlburt, Mrs. Jerome, M.

Hirsch, Mrs. L. H., M.

Hicks, Martha and John, M.

Huested, Mrs. G. Ralph, M.

H., Miss, M.

H., Miss, M.

Howells, Mildred and William Dean (bas-relief), 275.

Homan, Mrs. Wm. Franklin, M.

Izzard, Mrs. Ralph (Alice De Lancey), 69.

Irving, Mrs. John, 88.

J. T., Miss, M.

Jennings, Mrs. Oliver G., 94.

Josephine, Empress, M.

Jones, Miss Beatrix, I8o.

James, Mrs. Henry Fairchild, M. 
Jones, Mrs. Gilbert E., $27 \mathrm{I}$.

Jones, Master Pembroke, M.

Key, Mrs. John R., M.

King, Rufus, 226.

Knapp, Mrs., I99.

Kuhn, Mary S., 227.

Kane, Mrs., g6.

Kinnicutt, Mrs. Francis P., I2.

Kemp, Mrs. Arthur, 10.

Key, Mrs. John R., M.

Ketcham, Mrs., I 48.

Kemble, John Philip, 157.

Kingdon, Mrs. Charles, M.

Kelikian, Mrs. Dikran, M.

Kingdın, Mrs., M.

Kırkland, Master Geo., M.

Le Moyne, Mrs. Wm. J., I6o.

Leu, Mrs. Charles M., M.

Lürnian, Mrs. Gustave, M.

Loring, Miss Louisa P., M.

Leidy, Mr. Joseph, M.

Leonard, Child of Mr. and Mrs. Arthur, M.

La Farge, Florence and Margaret, M.

Lydia ; a portrait, M.

Little, Mrs. Arthur W., M.

L. C. F., Master, I5.

Little, Mrs. Arthur W., 47.

Livingston, Mrs. Henry Gilbert, M.

Lowery, Miss Milly, M.

Livingston, Johnston, 82.

Lady with a Muff, 205. 
Lady as a Mythological Personage, 272.

Lawrence, Augustin Hicks, 224.

Lawrence, John, 232.

Lawrence, Mrs. John, 23I.

Lea, Mrs. Thomas (née Sally Shippen), 225.

Lincklaen, Mrs., I 8 I.

Litchfield, Miss Marian, M.

Longstreth, Miss H. G., M.

Louise Matilda of Bayrout, M.

Litchfield, Mrs. Edward, M.

Litchfield, Miss, M.

Lady Playing Harp, 5 I6.

Mowbray, Mrs., 165.

Mackay, Mrs. Clarence H., 38.

Montant, Mrs. Jules A., $4 \mathrm{I}$.

Mackay, John W., 48.

Morton, Mrs. Levi P., M.

Morton, Miss, $8 \mathrm{r}$.

Moeran, Mrs., 9 I.

Morny, Duchesse de, 92.

Morgan, J. Pierpont, I 33 .

Morton, Miss Helen, I35.

Morton, Miss Lena, I 36 .

Morse, Mrs. Robt. M., I 30.

Marquand, Mrs. Isaac, I Io.

Marlborough, Duchess of, M.

M. T., Miss, M.

Myers, Miss Sylvia, M.

M D ., M.

Mrs. H. as a "Geisha Girl," M.

My Color Bearer, M.

Morrogh, Mrs. Charles, M. 
Marquand, Mr. Henry G., M.

Myrick, Miss, M.

M., Miss M.

Mrs. H. G. P., and Child, M.

Medici, Catherine de', M.

Millspaugh, Miss, M.

Mercer, Mr., M.

Mother and Child, M.

Mercer, Mrs. J. R., M .

Meeker, Miss, M.

M., Mrs., M.

North, Alfred Barham, M.

Norrie, Children of Mr. and Mrs. A. Lanfear, M.

Nichols, Mrs. Chas. McKinstry, M.

Ogden, Miss Polly, 30.

Oothout, Mrs. Henry, M.

Oldberg, Miss Olga, M.

Oldberg, Master Virgil, M.

Opp, Miss Julia, M.

Oldberg, Miss Olga, M.

Oldberg, Master Virgil, M.

Palmer, Mrs. Potter, 265.

Pease, Mrs., 247.

Pierson, Miss, 252.

Pierson, Mr. and Mrs. T. Green (I8I2), M.

Poor, Mrs. Henry W., M.

Powell, Harford Willing Hare, 259.

Postley, Commodore, M.

Perry, Commodore, 146.

P. K., Mrs., I8. 
Poor, Mrs. Henry W., I9.

Portrait of Cynthia, 23.

Palmer, Mrs. Walter L., 59.

Profile of Woman, 99.

Polk, Dr. W. M., 93.

Portrait of Mr. Musters, 20I.

Portrait Study of a Cardinal, M.

Powel, Robt. J. Hare, Children of, M.

Potter, Bishop, M.

Portrait of Three Young Ladies, 5 I7.

R., W. S., 50.

Randolph, Cora, I2 I.

Reade, Sir John, Bart., 273.

Redmond, Johnston and Roland L., 54.

Redmond, Mrs. G., 53.

Reid, Miss J. C., I20.

Reynal, V. C., I39.

Rittenhouse, Miss, M.

Rogers, Master Herman Livingston, M.

Roosevelt, Mrs. James, M.

Rosamond, S., 230.

Russell, Mrs. William Hamilton, Ioo.

Russell, Mrs. C. H., and Children, I I9.

Russia, Katherine of, M.

Rutter, Mrs. V. E., M.

Roche, Mrs. Burke, 89.

Robinson, Miss Florence, 32.

Rodewald, Mrs., M.

Richard, Mrs. Oscar L., 26r.

Richards, Nathaniel (I825), M.

Ricketts, Mr. Wm., M.

Reproduction of His Royal Highness, Prince of Wales, 522 . 
Seligman, Jesse, M.

Slocum, Mrs. Margaret Presian Jermain, M.

Stevenson, Mrs. S. M., M.

Sachini, I96.

Sage, Russell, 234.

Sage, Mrs. Russell, M.

Sallé, Mme., a Dancer of the First Empire, M.

Savidge, Dr. Eugene Coleman, 60.

Seymour, Mrs. H. T. (née Damrosch), I 37.

Sister of Rev. John Wesley Brown, D.D.. M,

Sproull, "Major," 270.

Stevenson, Robert Louis (bas-relief), 274.

Starr, Mrs. Louis, M.

Stone, Mrs. W. A., 268.

Strong, Master Frederick, Jr., M.

Smith, Mr. H. Y. S., M.

Stewart, Mrs. F. St. M., M.

Smith, Mrs. Clinch, I 2.

Strong, Edward A., I 3 I.

Sherman, Mrs. W. Watts, M.

Sherman, Mrs. W. Watts, 168.

Sherman, Miss G., I 70.

Sherman, Miss I., I69.

Schieffelin, Mr. George R., M.

Steiner, Mrs. Louis, and Sons, 73.

St. John, Rev. Canon, of Gloucester Cathedral, 78.

Sloane, Mrs. Samuel, Jr., I05.

Schermerhorn, Miss, 95.

Stanhope, Lady, 197.

Stevens, Mrs. Chấs. Emory, I9I.

Stirling, Lord, 200.

Siddons, Mrs., 24I.

Spencer, Mrs. Lorillard, 523. 
Taylor, Miss Edith Moore, M.

Taylor, Miss Marian, M.

Thompson, Mrs. Wm. R., 56.

Thompson, Wm. R., 57 .

Town, I74.

Truax, Mrs., I I 3.

Tilghman, Mrs. Ben, M.

Trenholm, Mrs. Alice, M.

Trowbridge, Miss, M.

Tower, Miss, M.

Talmage, E. T. H., Jr., M.

Thill, Miss Constance, M.

The Cigarette Girl, 267.

The Woman in White, 254.

Torrey, Mme. G. B., 237.

Tyler, Mrs. M. A., 520.

Terrell, Mrs. Herbert Leslie, 52 I.

Telfer, Mrs., M.

Van Buren, President, I43.

Venetian Senators (from the Riccardi Palace), 240.

Vicq, Baron de (copy of Rubens' portrait of, in the Louvre), $M$.

Van Ingen, Mrs. McLane, 527.

Van Buren, Miss Mary, M.

Vansittart, Lady, 5 I4.

Waters, Miss, M.

Winthrop, Mrs. Edgerton L., Jr., M.

Walch, Miss Ellen, M.

West, Benjamin, M.

Wheeler, Mrs. E. Van Zandt, M.

Wheeler, Mrs. J. Harry, M. 
Whitney, Mrs. W. C., M.

Wiles, Mr. and Mrs. L. M., 255.

Wolfe, Miss Elsie de, 2I.

Wilder, Miss, M.

Wilder, Miss Mella, M.

Winslow, Miss, 47.

Ward, Mr. Beverly, M.

Wilder, S. V. S. (I819), M.

Wilder, Mrs. S. V. S. (1819), M.

Wood, Mrs. Geo. Edw., M.

Waddilove, Miss, 202.

Walcott, Dr., as Peter Pindar, I77.

Wertheimer, 220.

Whestler, J. McN., I62.

Willett, Mrs. Ralph, 207.

Whittemore, Mrs. Charles, M.

W., Mrs., M.

Woodsworth, Mrs. Lydia S., 58.

Woman with Guitar, 97.

Wells, Master John, IO2.

Wysong, Mrs. J. C., I.

Wilson, Mrs. Orme, 526.

Wurtemburg, Princess of, 515 .

Wilde, Mrs. Harold W., M.

Walch, Miss Ellen, M. 


\section{LIST OF ARTISTS CONTRIBUTING TO THE PRESENT EXHIBITION.}

Alexander, J. W., Paris.

Anderson, A. A., 93 Fifth Avenue.

Arter, J. Chas., I9 West 2 Ist Street.

Baker, Martha,

Beaux, Cecilia, I 7 ro Chestnut Street, Philadelphia.

Beckwith, J. Carroll, 58 West 57th Street.

Bell, Edw. A., 226 Central Park, South.

Benson, Frank W., Salem, Mass.

Blenner, Carl J., 58 West 57th Street.

Brooks, Maria, 58 West 57 th Street.

Campbell, Georgine, 922 Madison Avenue.

Carpenter, K. Holston, 98I Madison Avenue.

Champney, J. Wells, 96 Fifth Avenue.

Chase, Wm. M., 57 West 57th Street.

Collins, Alfred Q., 883 Seventh Avenue.

Cox, Kenyon, 75 West 55 th Street.

Curran, Chas. C., I6 West 6 Ist Street.

De Luce, Percival, 52 East 23d Street.

Dewey, Chas. Melville, 222 West 23d Street.

Dewing, T. W., I42 East I8th Street.

Emmet, Lydia Field, New Rochelle, N. Y. 
Finocchiaro, Francesco Paolo, I 93 Broadway.

Flagg, Jared B., 37 West 22d Street.

Fowler, Frank, 106 West 55 th Street.

Frazier, Kenneth, 4I Union Square.

Funk, W. H., 4I Union Square.

Hardie, Robert Gordon, Carnegie Hall.

Heller, E. M., 58 West 57 th Street.

Herter, Albert, 84I Madison Avenue.

Heustis, Louise L., 53 East 56th Street.

Hughes, Edward, London.

Huntington, D., 49 East 20th Street.

Hyde, Wm. H., I05 East 6rst Street.

Isham, Samuel, 58 West 57th Street.

Johnson, Eastman, 65 West $55^{\text {th Street. }}$

Keith, Dora W., I24 East 27th Street.

Kendall, Wm. Sargent, 212 West 42d Street

Ketcham, Susan M., Carnegie Hall.

Lambert, John, Jr., Philadelphia.

Lippincott, Wm. H., 256 West 84th Street.

Lumsdon, E. Christine, 939 Eighth Avenue.

Millspaugh, Miss,

Morgan, Miss Jane, Bard Avenue, Staten Island.

Mowbray, H. Siddons, 66 West I I th Street.

Munzy, Geo. C., 627 Madison Avenue.

Parsons, Orrin S., I I 5 East 23d Street.

Pennington, Harper, $5 \mathrm{I}$ West Ioth Street.

Poore, H. R., Orange, N. J.

Porter, Benj. C., 22 West IIth Street. 
Rice, Wm. M. J., 55 West 33d Street.

Sargent, John S., 33 Tite Street, Chelsea, S. W., London, England.

Shepley, Annie B., 96 Fifth Avenue.

Sherwood, Rosina Emmet, 3 Io West 88th Street.

Stewart, Jules, Paris.

Story, Julian, Paris.

Strickland, C. Hobart, 146 West 55 th Street.

Tack, Augustus Vincent, Van Dyck Studio.

Telfer, J. C.

Thorne, Wm., I 54 West 55 th Street.

Tillinghast, Mary C., 3 Washington Square, N.

Torrey, Geo. Burroughs, 39I Fifth Avenue.

Troubetskoy, Prince Pierre, Washington, D. C.

Turner, Shirley,

Ury, A. Muller, 58 West 57 th Street.

Vinton, Frederic P., 247 Newbury Street, Boston.

Waring, E. B., Newport, R. I.

Weir, J. Alden, I I East 12th Street.

Whitman, Mrs. H., I84 Boylston Street, Boston.

Wilde, Ida M.,

Wiles, Irving R., 106 West $55^{\text {th }}$ Street.

Williams, Frederick B., Glen Ridge, N. J.

Wores, Theodore, 2 West 33d Street. 


\section{IMMAN@ZORE}

IMPORTERS OF

\section{Fine China, Glass, and Pottery,}

\section{5th Ave. and 3oth St., New York.}

FIFTH AVENUE TRUST COIMPANY

43d STREET AND FIFTH AVENUE

Capital and Surplus, $\$ 1,000,000$

Money received on deposit subject to check as in any bank, and interest allowed on balances.

OFFICERS

LEVI P. MORTON,-President

W.M. D. SLOANE, Vice-President CECIL D. LANDALE, 2d Vice-President

HERBERT H. SWASEY, Secretary JAMES M. PRATT, Assistant Secretary

TRUSTEES

S. D. Babcock, August Belmont, E. J. Berwind, Chauncey M. Depew, C. D. Dickey, E. T. Gerry, Marcellus Hartley, G. G. Haven, Jos. C. Hendrix, J. H. Hyde, A. Iselin, Jr., A. D. Julliard, D. H King, Jr., Daniel Lord, Levi P. Morton, Wm. D. Sloane, Frank Tilford, Wm. C. Whitney.

BUDD, MADISON SQUARE, WEST,

\section{Holiday Presents for Men.}

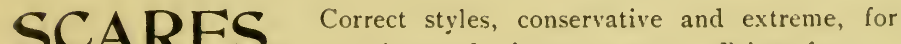

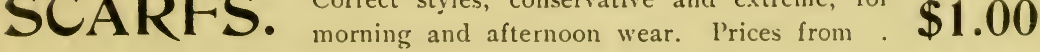

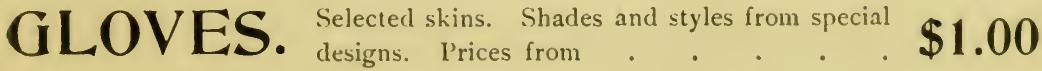

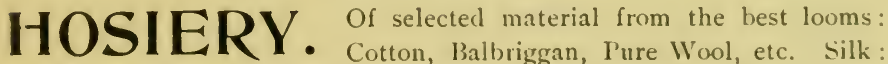
solid colors, embroidered and open work. I'rices from . . . 50 5 .

Handkerchiefs, Braces, Umbrellas, Canes, Bath and Dressing Gowns, etc.

SOLE AGENT FOR THE CELEBRATED . .
COON'S FUR AND SILK GLOVES. 


\section{Art Gifts for Christmas.}

An unusually attractive display of all styles and sizes, ready for immediate delivery at the art rooms of

\section{BERLIN PHOTOGRAPHIC COMPANY,}

Fine Art Publishers,

I4 EAST 23D STREET, $==\quad=\quad=$ NEW YORK. (Madison Square, South.)

\section{THE}

LINCOLN NATIONAL BANK

оि тив

\section{CITY OF NEW YORK}

32=42 East 42d Street

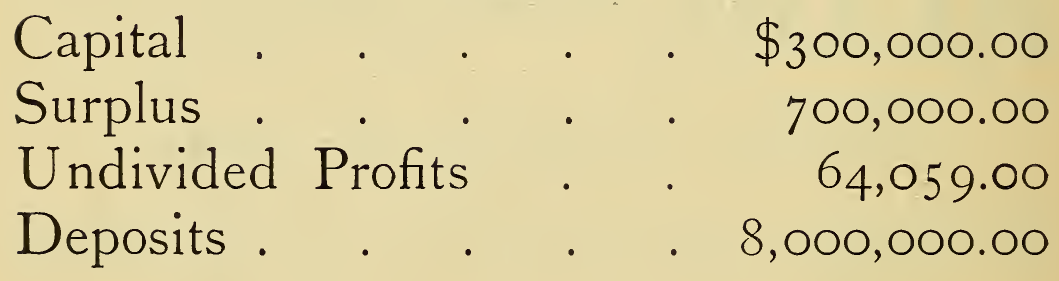

THOMAS L. JAMES, President

EDWARD V. W. ROSSITER, Vice-President

J. W. LAYNG, 2D Vice-President

WILLIAM T. CORNELL, CashIER

TALCOTT C. VAN SANTVOORD, Asst. Cashier

Personal, Family and Business Accounts Solicited 


\section{SEASON 1898-1899}

\section{Sommer's}

Red, Blue, White Hungarian Bands,

And Newly Arrived Black Hussars.

THE RUSSIA COURT ORCHESTRA,

THE ROUMANIAN BAND,

THE FLORENTINE MANDOLIN QUINTETTE.

For Concerts, Musicales, Weddings, Balls, Cotillons, Dancing Parties, Fairs,

Churches, etc.

\section{REFERS BY PERMISSION}

Mr. Fohn Facob Astor, Mrs. Timothy L. Woodruff, Mr. H. McK. Twombly', Mrs. George Griswold,
Mr. Pierre Lorillard, Fr., Mrs. Elliot F. Shephard, Mr. \& IIrs. Grenville Kane, Mr. \& Mrs. George Gould,

Mrs. Grower Cleveland.

\section{ENGAGED FOR THE SEASON AT}

The Waldorf-Astoria, Logerot-Flouret, Walton, and Lakewood Hotels, TUXedo, and N. Y. Yacht Clubs, etc.

For Terms, particulars, dates, etc., apply to

$$
\begin{aligned}
& \text { Leo Sommer, P/.D., } \\
& 57 \text { East r2Ist, Street, } \\
& \text { New York. }
\end{aligned}
$$

Telephone Call, 266 harlem.

THE BEST DANCE-MUSIC PLAYERS IN THE WORLD. 
Cor. 28th St.

Attention is invited to our perfect facilities for the COPYING AND ENLARGEMENT OF PHOTOGRAPHS

\section{BY THE CARBON PROCESS}

in any desired tint in monotone from an ordinary cabinet photograph, and, as the work is done photographically without alteration, the likeness can be guaranteed absolutely.

Our facilities for executing the work are not excelled in any part of the world.

Prices are the same as Sittings Direct from Life.

Enlarged portraits made from small photographs by the Carbon Process are superior to Crayons or ordinary photographic enlargements, and, being absolutely durable and unalterable in color, will give a degree of satisfaction unattainable by any other process.

Specimens of this work can be seen at our Studio.

Visitors to Studio are always welcome.

Portraits Direct from Life in any size or style of finish. Ideal Results. THE MOST PERFECT
ASSORTMENT OF

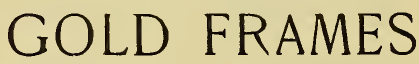

IN AMERICA.

Special Designs in Paris-Made Metal Frames for Miniatures and Photographs in very large variety. Inspection Invited.

DAVIS \& SANFORD, 246 Fifth Ave., cor. 28 th St., N. Y.

THE FIRST "BANK OF DEPOSIT" THAT PROVIDED SEPARATE BANKING FACILITIES FOR WOMEN, A.D. I869.

JOHN L. RIKER, PRES'T.
THE SE
SECOND
NATIONAL BA

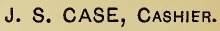

OF THE CITY OF NEW YORK,

FIFTH AVENUE, CORNER 23d STREET

(Under Fifth Avenue Hotel), AND

FIFTH AVENUE SAFE DEPOSIT COMPANY, CAPITAL, $\$ 300,000$. SURPLUS AND UNDIVIDED PROFITS, $\$ 700,000$. Fifth Avenue, corner 23d Street (Fifth Avenue Hotel).

LADIES'

AND

CHILDREN'S

COSTUMES

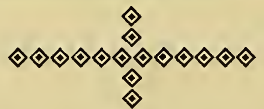

HATS

AND

JAQUETS

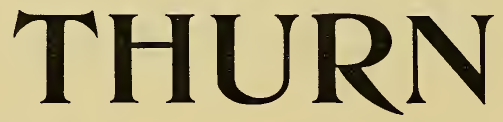

\section{Bellevue Avenue} NEWPORT

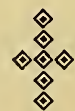

426 Fifth Avenue NEW YORK 
A. D. VORCE,

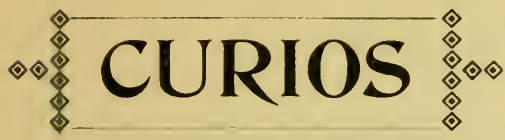

ORIENTAL

ANTIQUES,

353 Fifth Avenue,

\section{Corner
$3_{4}$ th Street,}

Opposite Waldorf-Astoria.

\section{AIMÉ DUPONT}

\section{Parisian Artist}

\section{and Photographer}

574 Fifth Ave.,

\section{NEW YORK}

Decorated several times by European Sovereigns, in recognition of artistic merit.

Flattering testimonials from the most eminent artists of the day. Gold medal Universal Exposition, Paris. Unique medal for good taste Vienna Exposition.

\section{G. A. RECHLIN \& CO,}

\section{Maker of Men's Garments}

220 Fifth Avenue, near 26th Street,

NEW YORK

\section{Riding Breeches}

Cut by G. A. Rechls, for World's Columbian Exposition, IS93, received Medals and Highest Awards.

NOVELTIES IN IMPORTED RICH FABRICS FOR THE PRESENT SEASON 


\section{HA ANS'}

RESTAURANT

N. E. Cor. $75^{\text {th }}$ St. and Columbus Ave.,

is the only first-class establishment on the West side, and in many respects the most unique in the city.

In its artistic appointments, in the excellence of its cuisine, and in the quiet perfection of its service it is an ideal place for After-Dinner and Theatre Parties, and as such it particularly appeals to the residents in that vicinity.

The menu is one of unusual interest. It embraces every delicacy known to the culinary art. A specialty is made of shellfish and game. Everything is moderately priced—so much so as often to incite both surprise and comment. There are private dining-rooms, and music every evening. 


\title{
Blakeslee Gallery
}

Fifth Ave. and 34th St.

\author{
(4) \\ EXHIBITION \\ OF \\ Early English \\ Flemish and Dutch
}

PAINTINGS

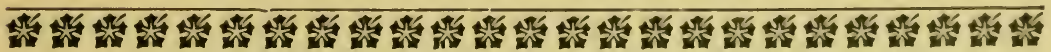




\section{ST. JOHN'S GUILD}

OF THE CITY OF NEW YORK.

A WHOLLY NON-SECTARIAN ORGANIZATION FOR THE RELIEF OF THE SICK CHILDREN OF THE POOR OF THE CITY OF NEW YORK, WITHOUT REGARD TO CREED, COLOR, OR NATIONALITY.

IN THIS WORK IT MAINTAINS :

\section{THE FLOATING HOSPITAL,}

NEW YORK HARBOR;

THE SEASIDE HOSPITAL,

NEW DORP, S. I. ;

\section{THE CHILDREN'S CITY HOSPITAL,}

Temporarily suspended;

SPECIAL RELIEF AND NURSING,

For ChILdREN in their Homes.

Temporarily suspended.

OFFICERS AND TRUSTEES.

W. L. STRONG, President.

WILLIAM SHERER, Ist Vice-President.

D. HUNTER MCALPIN, Jr., M.D., $2 d$ Vice-President.

JOHN P. FAURE,

Secretary.

WILLIAM H. WILEY,

JOHN W. WEED,

JOHN P. FAURE,

JOHN T. IJAMS,

WILLIAM BROOKFIELD, LLOYD I. SEAMAN,

JOHN CLAFLIN,

WM. HILDRETH FIELD,

ROBERT H. GREENE, M.D.,

WILLIAM F. KING,

HENRY MARQUAND,

WILLIAM R. WARREN,
WILLIAM R. WARREN Treasurer.

TRUSTEES.

FRANKLIN BURDGE,

W. W. FLANAGAN,

WALTER STANTON,

JAMES E. CHANDLER,

J. HENRY FRUITNIGHT, M.D.,

W. L. STRONG,

DILLON BROWN, M.D.,

D. HUNTER MCALPIN, JR., M.D.,

WILLIAM SHERER,

WILLIAN T. WARDWELL,

CHARLES O. BREWSTER,

WILLIAM H. BURR.

DUFF G. MAYNARD,

General Agent,

Office, 5Oi Fifth Avenue, New York. 


\section{STRONG, STURGIS \& CO.}

\section{Broad Street}

WM. E. STRONG

F. K. STURGIS

GEO. G. HAVEN, Jr.

NEW YORK

S. L. CRONWELL 
WM. LANMAN BULL

Member of N. Y. Stock Exchange
FREDERIC VAN LENNEP

Member of N. Y. Produce Exchange

\section{EDWARD SWEET \& CO.}

Bankers and Brokers

No. 38 Broad Street

NEW YORK

Private Wires to Boston, Philadelphia, and Washington 


\section{CLARK, DODGE \& CO.}

\section{Bankers}

No. 5I Wall Street

NEW YORK 


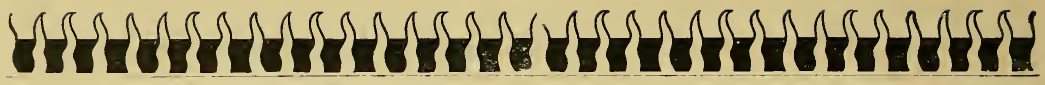

ROGERS \& GOULD

\section{Bankers and Brokers}

- 7I Broadway-

NEW YORK 


\title{
H. B. HOLLINS \& CO.,
}

BANKERS,

NEW YORK.

\begin{abstract}
ISSUE LETTERS OF CREDIT FOR TRAVELLERS AVAILABLE IN ALL PARTS OF THE WORLD.
\end{abstract}

ACT AS FINANCIAL AND TRANSFER AGENTS FOR CORPORATIONS.

EXECUTE ORDERS FOR THE PURCHASE AND SALE OF STOCKS, BONDS, AND ALL CLASSES OF INVESTMENT SECURITIES ON THE NEW YORK STOCK EXCHANGE, AS WELL AS ON THE PRINCIPAL EXCHANGES OF EUROPE AND THE UNITED STATES.

DRAW BILLS OFं EXCHANGE AND MAKE CABLE TRANSFERS ON THE PRINCIPAL CITIES OF EUROPE, THE WEST INDIES, CENTRAL AND SOUTH AMERICA.

MAKE COLLECTIONS IN ALL PARTS OF THE WORLD. 


\section{Tknickerbocker}

MAIN OFFICE :

234 Fifth Ave., cor. 27th St.
BRANCH OFFICE :

66 Broadway.

\title{
CAPITAL, $\$ 1,000,000$
}

\author{
DESIGNATED A LEGAL DEPOSITARY FOR
}

THE STATE OF NEW YORK,

THE GREATER NEW YORK,

\section{and for MONEYS PAID INTO COURT.}

Deposits Received Subject

to Check.
Interest Allowed on

Time Deposits.

Safe Deposit Boxes to Rent in Fire- and Burglar-Proof Vaults.

Authorized to act as Executor, Administrator, Guardian, Receiver, Registrar, Transfer, and Financial Agent, and accepts other Trusts in conformity with the laws of any State or of the United States.

\section{SiDanagement of IEstates a \$pecialty, for wbicb tReal IEstate IDortgages are always kept on bano.}

SEPARATE DEPARTMENT WITH SPECIAL FACILITIES FOR LADIES BUSINESS AND PERSONAL ACCOUNTS SOLICITED.

CHARLES T. BARNEY, Acting President. JOS. T. BROWN, 2d Vice-President. FREDK. L. ELDRIDGE, Sec'y \& Treas. J. HENRY TOWNSEND, Ass't Sec'y. ALFRED B. MACLAY, Ass't Treas.

BOARD OF DIRECTORS.

JOS. S. AUERBACH, CHARLES T. BARNEY, ANDREW H. SANDS, HON. E. V. LOEW, Charles F. Watson, C. Lawrence Perkins, AMZI L. BARBER,
HARRY B. HOLLINS, A. Foster Higgins, JAMES H. BRESLIN, Henry F. Dimock, FREDERICK G. BOURNE, AlFRED L. White, CHARLES T. COOK,
JACOB HAys,

HENRY W. T. MALI, I. TOWNSEND BURDEN, CHARLES R. FIINT, JOHN MAGEE, HeNRy A. MORGaN, HENRY C. BERLIN. 